\begin{tabular}{|c|c|}
\hline Title & Lightlike flat geometry of spacelike submanifolds in Lorentz-Minkowski space \\
\hline Author(s) & Izumiya, Shyuichi; Kasedou, Masaki \\
\hline Citation & $\begin{array}{l}\text { International Journal of Geometric Methods In Modern Physics, 11(5), } 1450049 \\
\text { https://doi.org/10.1142/S0219887814500492 }\end{array}$ \\
\hline Issue Date & 2014.05 \\
\hline Doc URL & http:/hdl.handle.net/2115/59114 \\
\hline Rights & $\begin{array}{l}\text { Electronic version of an article published as [International journal of geometric methods in modern physics, 11, 5, 2015, } \\
1450049 \text { [35 pages]] } \odot \text { [copyright World Scientific Publishing Company] }\end{array}$ \\
\hline Type & article (author version) \\
\hline File Information & IJGMMP11_1450049.pdf \\
\hline
\end{tabular}

Instructions for use 


\title{
Lightlike flat geometry of spacelike submanifolds in Lorentz-Minkowski space
}

\author{
Shyuichi IZUMIYA and Masaki KASEDOU*
}

January 24, 2014

\begin{abstract}
In this paper we investigate differential geometry on spacelike submanifolds in LorentzMinkowski space from the view point of contact with lightlike hyperplanes. It is called the lightlike flat geometry which has been well established for the codimension two case. In order to develop the theory for the general codimension case, we introduce the notion of codimension two spacelike canal submanifolds which is a main tool in this paper. We apply the theory of Lagrangian/Legendrian singularities to codimension two spacelike canal submanifolds and obtain the relation with the previous results on the codimension two case.
\end{abstract}

\section{Introduction}

In this paper we consider differential geometry on general spacelike submanifolds in LorentzMinkowski space. In the previous researches $[7,8,10,11,14]$, it has been recognized that the codimension two spacelike submanifolds have special meanings in differential geometry of spacelike submanifolds in Lorentz-Minkowski space. The lightlike geometry of spacelike submanifolds of codimension two has been developed in those articles which is a natural generalization of extrinsic differential geometry of hypersurfaces in Euclidean space and the horospherical geometry of hypersurfaces in Hyperbolic space [6]. If we consider general submanifolds in Euclidean space or Hyperbolic space, the canal hypersurface of the manifold is a useful tool for the study of extrinsic differential geometry on the manifold. The situation is rather complicated for spacelike submanifolds with general codimensions in Lorentz-Minkowski space comparing with the Euclidean space case or the Hyperbolic space case. The main difference from the Euclidean space case or the Hyperbolic space case is the fiber of the canal hypersurface of a spacelike submanifold is neither connected nor compact. However, we do not need the canal hypersurfaces for developing the lightlike geometry of spacelike submanifolds with general codimensions. According to the results of [11], we need codimension two spacelike canal submanifolds in Lorentz-Minkowski

*This work was partly supported by the JSPS International Training Program(ITP).

2010 Mathematics Subject classification:53C40, 58K05

Key Words and Phrases: Lorentz-Minkowski space, canal spacelike submanifold, lightcone Gauss maps, lightcone pedal hypersurface, Lagrangian/Legendrian singularities 
space. In order to define the notion of codimension two spacelike canal submanifolds, we arbitrary choose a timelike future directed unit normal vector field along a spacelike submanifold which always exists for an orientable spacelike submanifold (cf, [12, 13]). Then we construct the unit spherical normal bundle relative to the above timelike unit normal vector field. The codimension two spacelike canal submanifold is a spacelike embedding of this unit spherical normal bundle into Lorentz-Minkowski space. Since it is codimension two, we can apply the previous results for codimension two spacelike submanifolds. In [11], it has been investigated the lightlike flat geometry of spacelike submanifolds of codimension two in Lorentz-Minkowski space. The notion of lightcone Gauss maps and lightcone pedals of codimension two spacelike submanifolds play a principal role in the lightlike flat geometry. The singularities of those mappings, which are the points with vanishing lightcone Gauss-Kronecker curvatures, describe the contact of codimension two spacelike submanifolds with lightlike hyperplane.

In this paper we investigate the lightlike geometry of spacelike submanifolds in LorentzMinkowski space with general codimensions from the view point of the contact with lightlike hyperplanes. The natural connection between geometry and singularities relies on the basic fact that the contact of a submanifold with the models of the ambient space can be described by means of the analysis if the singularities of appropriate families of contact functions, or equivalently, of their associated Lagrangian/Legendrian maps. For the lightlike geometry the models are lightlike hyperplanes or lightcones. The lightlike flat geometry is the lightlike geometry which adopts lightlike hyperplanes as model hypersurfaces.

In $\S 3$ we briefly review some previous results on lightlike differential geometry of spacelike submanifolds which have been given in $[12,13]$. The lightcone Lipschitz-Killing curvature of spacelike submanifolds is one of the basic invariants. Lightcone height functions are defined in $\S 4$ and we investigate basic properties. Codimension two spacelike canal submanifolds are introduced in $\S 5$. We introduce the notion of lightcone Gauss maps and lightcone pedal hypersurfaces of codimension two spacelike canal submanifolds. Of course, it might depend on the choice of the future directed timelike unit normal vector field. However, we can show that these mappings are independent of the choice of the future directed unit normal vector field (cf., Proposition 5.2). Therefore, we can define suspended lightcone Gauss maps and suspended lightcone pedal hypersurfaces of the spacelike submanifold. In $\S 6$ we investigate the contact of spacelike submanifolds with lightlike hyperplanes. We investigate the detailed properties of contact with lightlike hyperplanes from the view point of the theory of Lagrangian/Legendrian singularities in $\S 7$ and $\S 8$. Finally, we consider the case that the future directed timelike unit normal vector field is parallel along the spacelike submanifold in $\S 9$. In this case we have more detailed properties. In $\S 10$ we consider spacelike curves in Lorentz-Minkowski 4-space as the simplest case of spacelike submanifolds with higher codimension. We consider the case of submanifolds lying in Euclidean space or Hyperbolic space in $§ 11$. In this case the lightlike flat geometry is the flat geometry in Euclidean space or the horospherical geometry in Hyperbolic space.

\section{Basic concepts}

We introduce in this section some basic notions on Lorentz-Minkowski $n+1$-space. For basic concepts and properties, see [17].

Let $\mathbb{R}^{n+1}=\left\{\left(x_{0}, x_{1}, \ldots, x_{n}\right) \mid x_{i} \in \mathbb{R}(i=0,1, \ldots, n)\right\}$ be an $n+1$-dimensional cartesian space. For any $\boldsymbol{x}=\left(x_{0}, x_{1}, \ldots, x_{n}\right), \boldsymbol{y}=\left(y_{0}, y_{1}, \ldots, y_{n}\right) \in \mathbb{R}^{n+1}$, the pseudo scalar product 
of $\boldsymbol{x}$ and $\boldsymbol{y}$ is defined by $\langle\boldsymbol{x}, \boldsymbol{y}\rangle=-x_{0} y_{0}+\sum_{i=1}^{n} x_{i} y_{i}$. We call $\left(\mathbb{R}^{n+1},\langle\rangle,\right)$ Lorentz-Minkowski $n+1$-space. We write $\mathbb{R}_{1}^{n+1}$ instead of $\left(\mathbb{R}^{n+1},\langle\rangle,\right)$. We say that a non-zero vector $\boldsymbol{x} \in \mathbb{R}_{1}^{n+1}$ is spacelike, lightlike or timelike if $\langle\boldsymbol{x}, \boldsymbol{x}\rangle>0,\langle\boldsymbol{x}, \boldsymbol{x}\rangle=0$ or $\langle\boldsymbol{x}, \boldsymbol{x}\rangle<0$ respectively. The norm of the vector $\boldsymbol{x} \in \mathbb{R}_{1}^{n+1}$ is defined to be $\|\boldsymbol{x}\|=\sqrt{|\langle\boldsymbol{x}, \boldsymbol{x}\rangle|}$. We have the canonical projection $\pi: \mathbb{R}_{1}^{n+1} \longrightarrow \mathbb{R}^{n}$ defined by $\pi\left(x_{0}, x_{1}, \ldots, x_{n}\right)=\left(x_{1}, \ldots, x_{n}\right)$. Here we identify $\{\mathbf{0}\} \times \mathbb{R}^{n}$ with $\mathbb{R}^{n}$ and it is considered as Euclidean $n$-space whose scalar product is induced from the pseudo scalar product $\langle$,$\rangle . For a vector \boldsymbol{v} \in \mathbb{R}_{1}^{n+1}$ and a real number $c$, we define a hyperplane with pseudo normal $\boldsymbol{v}$ by

$$
H P(\boldsymbol{v}, c)=\left\{\boldsymbol{x} \in \mathbb{R}_{1}^{n+1} \mid\langle\boldsymbol{x}, \boldsymbol{v}\rangle=c\right\} .
$$

We call $H P(\boldsymbol{v}, c)$ a spacelike hyperplane, a timelike hyperplane or a lightlike hyperplane if $\boldsymbol{v}$ is timelike, spacelike or lightlike respectively.

We now define Hyperbolic $n$-space by

$$
H_{+}^{n}(-1)=\left\{\boldsymbol{x} \in \mathbb{R}_{1}^{n+1} \mid\langle\boldsymbol{x}, \boldsymbol{x}\rangle=-1, x_{0}>0\right\}
$$

and de Sitter $n$-space by

$$
S_{1}^{n}=\left\{\boldsymbol{x} \in \mathbb{R}_{1}^{n+1} \mid\langle\boldsymbol{x}, \boldsymbol{x}\rangle=1\right\}
$$

We define

$$
L C^{*}=\left\{\boldsymbol{x}=\left(x_{0}, x_{1}, \ldots, x_{n}\right) \in \mathbb{R}_{1}^{n+1} \mid x_{0} \neq 0,\langle\boldsymbol{x}, \boldsymbol{x}\rangle=0\right\}
$$

and we call it the (open) lightcone at the origin.

If $\boldsymbol{x}=\left(x_{0}, x_{1}, \ldots, x_{2}\right)$ is a non-zero lightlike vector, then $x_{0} \neq 0$. Therefore we have

$$
\widetilde{\boldsymbol{x}}=\left(1, \frac{x_{1}}{x_{0}}, \ldots, \frac{x_{n}}{x_{0}}\right) \in S_{+}^{n-1}=\left\{\boldsymbol{x}=\left(x_{0}, x_{1}, \ldots, x_{n}\right) \mid\langle\boldsymbol{x}, \boldsymbol{x}\rangle=0, x_{0}=1\right\} .
$$

We call $S_{+}^{n-1}$ the lightcone (or, spacelike) unit $n-1$-sphere.

For any $\boldsymbol{x}_{1}, \boldsymbol{x}_{2}, \ldots, \boldsymbol{x}_{n} \in \mathbb{R}_{1}^{n+1}$, we define a vector $\boldsymbol{x}_{1} \wedge \boldsymbol{x}_{2} \wedge \cdots \wedge \boldsymbol{x}_{n}$ by

$$
\boldsymbol{x}_{1} \wedge \boldsymbol{x}_{2} \wedge \cdots \wedge \boldsymbol{x}_{n}=\left|\begin{array}{cccc}
-\boldsymbol{e}_{0} & \boldsymbol{e}_{1} & \cdots & \boldsymbol{e}_{n} \\
x_{0}^{1} & x_{1}^{1} & \cdots & x_{n}^{1} \\
x_{0}^{2} & x_{1}^{2} & \cdots & x_{n}^{2} \\
\vdots & \vdots & \cdots & \vdots \\
x_{0}^{n} & x_{1}^{n} & \cdots & x_{n}^{n}
\end{array}\right|,
$$

where $\boldsymbol{e}_{0}, \boldsymbol{e}_{1}, \ldots, \boldsymbol{e}_{n}$ is the canonical basis of $\mathbb{R}_{1}^{n+1}$ and $\boldsymbol{x}_{i}=\left(x_{0}^{i}, x_{1}^{i}, \ldots, x_{n}^{i}\right)$. We can easily check that $\left\langle\boldsymbol{x}, \boldsymbol{x}_{1} \wedge \boldsymbol{x}_{2} \wedge \cdots \wedge \boldsymbol{x}_{n}\right\rangle=\operatorname{det}\left(\boldsymbol{x}, \boldsymbol{x}_{1}, \ldots, \boldsymbol{x}_{n}\right)$, so that $\boldsymbol{x}_{1} \wedge \boldsymbol{x}_{2} \wedge \cdots \wedge \boldsymbol{x}_{n}$ is pseudo orthogonal to any $\boldsymbol{x}_{i}(i=1, \ldots, n)$.

\section{Differential geometry on spacelike submanifolds}

In this section we introduce the basic geometrical framework for the study of spacelike submanifolds in Lorentz-Minkowski $n+1$-space analogous to the case of codimension two in [11]. Let $\mathbb{R}_{1}^{n+1}$ be a timelike oriented space. We choose $\boldsymbol{e}_{0}=(1,0, \ldots, 0)$ as the future timelike vector field. Let $\boldsymbol{X}: U \longrightarrow \mathbb{R}_{1}^{n+1}$ be a spacelike embedding of codimension $k$, where $U \subset \mathbb{R}^{s}$ $(s+k=n+1)$ is an open subset. We also write $M=\boldsymbol{X}(U)$ and identify $M$ and $U$ through 
the embedding $\boldsymbol{X}$. We say that $\boldsymbol{X}$ is spacelike if the tangent space $T_{p} M$ of $M$ at $p$ consists of spacelike vectors for any point $p \in M$. For any $p=\boldsymbol{X}(u) \in M \subset \mathbb{R}_{1}^{n+1}$, we have

$$
T_{p} M=\left\langle\boldsymbol{X}_{u_{1}}(u), \ldots, \boldsymbol{X}_{u_{s}}(u)\right\rangle_{\mathbb{R}}
$$

where $u=\left(u_{1}, \ldots, u_{s}\right)$. Let $N_{p}(M)$ be the pseudo-normal space of $M$ at $p$ in $\mathbb{R}_{1}^{n+1}$. Since $T_{p} M$ is a spacelike subspace of $T_{p} \mathbb{R}_{1}^{n+1}, N_{p}(M)$ is a $k$-dimensional Lorentzian subspace of $T_{p} \mathbb{R}_{1}^{n+1}$ (cf.,[17]). On the pseudo-normal space $N_{p}(M)$, we have two kinds of pseudo spheres:

$$
\begin{aligned}
N_{p}(M ;-1) & =\left\{\boldsymbol{v} \in N_{p}(M) \mid\langle\boldsymbol{v}, \boldsymbol{v}\rangle=-1\right\} \\
N_{p}(M ; 1) & =\left\{\boldsymbol{v} \in N_{p}(M) \mid\langle\boldsymbol{v}, \boldsymbol{v}\rangle=1\right\}
\end{aligned}
$$

so that we have two unit normal spherical normal bundles over $M$ :

$$
N(M ;-1)=\bigcup_{p \in M} N_{p}(M ;-1) \text { and } N(M ; 1)=\bigcup_{p \in M} N_{p}(M ; 1)
$$

We have the Whitney sum decomposition $T \mathbb{R}_{1}^{n+1} \mid M=T M \oplus N(M)$. Since $M=\boldsymbol{X}(U)$ is spacelike, $\boldsymbol{e}_{0}$ is a transversal future directed timelike vector field along $M$. For any $\boldsymbol{v} \in T_{p} \mathbb{R}_{1}^{n+1} \mid M$, we have $\boldsymbol{v}=\boldsymbol{v}_{1}+\boldsymbol{v}_{2}$, where $\boldsymbol{v}_{1} \in T_{p} M$ and $\boldsymbol{v}_{2} \in N_{p}(M)$. If $\boldsymbol{v}$ is timelike, then $\boldsymbol{v}_{2}$ is timelike. Let $\pi_{N(M)}: T \mathbb{R}_{1}^{n+1} \mid M \longrightarrow N(M)$ be the canonical projection. Then $\pi_{N(M)}\left(\boldsymbol{e}_{0}\right)$ is a future directed timelike normal vector field along $M$. So we always have a future directed unit timelike normal vector field along $M$ (even globally). We now arbitrarily choose a future directed unit timelike normal vector field $\boldsymbol{n}^{T}(u) \in N_{p}(M ;-1)$, where $p=\boldsymbol{X}(u)$. Therefore we have the pseudoorthonormal compliment $\left(\left\langle\boldsymbol{n}^{T}(u)\right\rangle_{\mathbb{R}}\right)^{\perp}$ in $N_{p}(M)$ which is a $(k-1)$-dimensional spacelike subspace of $N_{p}(M)$. We can also choose a pseudo-normal section $\boldsymbol{n}^{S}(u) \in\left(\left\langle\boldsymbol{n}^{T}(u)\right\rangle_{\mathbb{R}}\right)^{\perp} \cap N(M ; 1)$, then we have $\left\langle\boldsymbol{n}^{S}, \boldsymbol{n}^{S}\right\rangle=1$ and $\left\langle\boldsymbol{n}^{S}, \boldsymbol{n}^{T}\right\rangle=0$. We define a $(k-2)$-dimensional spacelike unit sphere in $N_{p}(M)$ by

$$
N_{1}(M)_{p}\left[\boldsymbol{n}^{T}\right]=\left\{\boldsymbol{\xi} \in N_{p}(M ; 1) \mid\langle\boldsymbol{\xi}, \boldsymbol{n}(p)\rangle=0\right\} .
$$

Then we have a spacelike unit $(k-2)$-spherical normal bundle over $M$ with respect to $\boldsymbol{n}^{T}$ defined by $N_{1}(M)\left[\boldsymbol{n}^{T}\right]=\bigcup_{p \in M} N_{1}(M)_{p}\left[\boldsymbol{n}^{T}\right]$. Then we have $T_{(p, \xi)} N_{1}(M)\left[\boldsymbol{n}^{T}\right]=T_{p} M \times T_{\xi} N_{1}(M)_{p}\left[\boldsymbol{n}^{T}\right]$.

For any future directed unit normal $\boldsymbol{n}^{T}$ along $M$, we arbitrary choose the unit spacelike normal vector field $\boldsymbol{n}^{S}$ with $\boldsymbol{n}^{S}(u) \in N_{1}(M)_{p}\left[\boldsymbol{n}^{T}\right]$ (i.e., $\left\langle\boldsymbol{n}^{S}, \boldsymbol{n}^{S}\right\rangle=1,\left\langle\boldsymbol{n}^{S}, \boldsymbol{n}^{T}\right\rangle=0$ ), where $p=$ $\boldsymbol{X}(u)$. We call $\left(\boldsymbol{n}^{T}, \boldsymbol{n}^{S}\right)$ a future directed orthonormal pair. Clearly, the vectors $\boldsymbol{n}^{T}(u) \pm \boldsymbol{n}^{S}(u)$ are lightlike. Here we choose $\boldsymbol{n}^{T}+\boldsymbol{n}^{S}$ as a lightlike normal vector field along $M$. We define a mapping $\mathbb{L} \mathbb{G}\left(\boldsymbol{n}^{T}, \boldsymbol{n}^{S}\right): U \longrightarrow L C^{*}$ by $\mathbb{L} \mathbb{G}\left(\boldsymbol{n}^{T}, \boldsymbol{n}^{S}\right)(u)=\boldsymbol{n}^{T}(u)+\boldsymbol{n}^{S}(u)$. We call it the lightcone Gauss image of $M=\boldsymbol{X}(U)$ with respect to $\left(\boldsymbol{n}^{T}, \boldsymbol{n}^{S}\right)$. We also define a mapping $\widetilde{\mathbb{L} G}\left(\boldsymbol{n}^{T}, \boldsymbol{n}^{S}\right): U \longrightarrow S_{+}^{n-1}$ by $\widetilde{\mathbb{L G}}\left(\boldsymbol{n}^{T}, \boldsymbol{n}^{S}\right)(u)=\boldsymbol{n}^{T}\left(\widetilde{u)+\boldsymbol{n}^{S}}(u)\right.$ which is called the lightcone Gauss map of $M=\boldsymbol{X}(U)$ with respect to $\left(\boldsymbol{n}^{T}, \boldsymbol{n}^{S}\right)$. Under the identification of $M$ and $U$ through $\boldsymbol{X}$, we have the linear mapping provided by the derivative of the lightcone Gauss image $\mathbb{L} \mathbb{G}\left(\boldsymbol{n}^{T}, \boldsymbol{n}^{S}\right)$ at each point $p \in M$,

$$
d_{p} \mathbb{L} \mathbb{G}\left(\boldsymbol{n}^{T}, \boldsymbol{n}^{S}\right): T_{p} M \longrightarrow T_{p} \mathbb{R}_{1}^{n+1}=T_{p} M \oplus N_{p}(M) .
$$

Consider the orthogonal projection $\pi^{t}: T_{p} M \oplus N_{p}(M) \rightarrow T_{p}(M)$ and define

$$
d_{p} \mathbb{L} \mathbb{G}\left(\boldsymbol{n}^{T}, \boldsymbol{n}^{S}\right)^{t}=\pi^{t} \circ d_{p}\left(\boldsymbol{n}^{T}+\boldsymbol{n}^{S}\right) .
$$


We call the linear transformation $S_{p}\left(\boldsymbol{n}^{T}, \boldsymbol{n}^{S}\right)=-d_{p} \mathbb{L} \mathbb{G}\left(\boldsymbol{n}^{T}, \boldsymbol{n}^{S}\right)^{t}$ of $T_{p} M$, the $\left(\boldsymbol{n}^{T}, \boldsymbol{n}^{S}\right)$ shape operator of $M=\boldsymbol{X}(U)$ at $p=\boldsymbol{X}(u)$. The eigenvalues of $S_{p}\left(\boldsymbol{n}^{T}, \boldsymbol{n}^{S}\right)$, denoted by $\left\{\kappa_{i}\left(\boldsymbol{n}^{T}, \boldsymbol{n}^{S}\right)(p)\right\}_{i=1}^{s}$, are called the lightcone principal curvatures with respect to $\left(\boldsymbol{n}^{T}, \boldsymbol{n}^{S}\right)$ at $p=\boldsymbol{X}(u)$. Then the lightcone Lipschitz-Killing curvature with respect to $\left(\boldsymbol{n}^{T}, \boldsymbol{n}^{S}\right)$ at $p=\boldsymbol{X}(u)$ is defined by

$$
K_{\ell}\left(\boldsymbol{n}^{T}, \boldsymbol{n}^{S}\right)(p)=\operatorname{det} S_{p}\left(\boldsymbol{n}^{T}, \boldsymbol{n}^{S}\right) .
$$

We say that a point $p=\boldsymbol{X}(u)$ is an $\left(\boldsymbol{n}^{T}, \boldsymbol{n}^{S}\right)$-umbilical point if all the principal curvatures coincide at $p=\boldsymbol{X}(u)$ and thus $S_{p}\left(\boldsymbol{n}^{T}, \boldsymbol{n}^{S}\right)=\kappa\left(\boldsymbol{n}^{T}, \boldsymbol{n}^{S}\right)(p) 1_{T_{p} M}$, for some function $\kappa$. We say that $M=\boldsymbol{X}(U)$ is totally $\left(\boldsymbol{n}^{T}, \boldsymbol{n}^{S}\right)$-umbilic if all points on $M$ are $\left(\boldsymbol{n}^{T}, \boldsymbol{n}^{S}\right)$-umbilic.

We deduce now the lightcone Weingarten formula. Since $\boldsymbol{X}_{u_{i}}(i=1, \ldots s)$ are spacelike vectors, we have a Riemannian metric (the lightcone first fundamental form ) on $M=\boldsymbol{X}(U)$ defined by $d s^{2}=\sum_{i=1}^{s} g_{i j} d u_{i} d u_{j}$, where $g_{i j}(u)=\left\langle\boldsymbol{X}_{u_{i}}(u), \boldsymbol{X}_{u_{j}}(u)\right\rangle$ for any $u \in U$. We also have a lightcone second fundamental invariant with respect to the normal vector field $\left(\boldsymbol{n}^{T}, \boldsymbol{n}^{S}\right)$ defined by $h_{i j}\left(\boldsymbol{n}^{T}, \boldsymbol{n}^{S}\right)(u)=\left\langle-\left(\boldsymbol{n}^{T}+\boldsymbol{n}^{S}\right)_{u_{i}}(u), \boldsymbol{X}_{u_{j}}(u)\right\rangle$ for any $u \in U$. By the similar arguments to those in the proof of [11, Proposition 3.2], we have the following proposition.

Proposition 3.1 We choose a pseudo-orthonormal frame $\left\{\boldsymbol{n}^{T}, \boldsymbol{n}_{1}^{S}, \ldots, \boldsymbol{n}_{k-1}^{S}\right\}$ of $N(M)$ with $\boldsymbol{n}_{k-1}^{S}=\boldsymbol{n}^{S}$. Then we have the following lightcone Weingarten formula with respect to $\left(\boldsymbol{n}^{T}, \boldsymbol{n}^{S}\right)$

(a) $\mathbb{L} \mathbb{G}\left(\boldsymbol{n}^{T}, \boldsymbol{n}^{S}\right)_{u_{i}}=\left\langle\boldsymbol{n}_{u_{i}}^{S}, \boldsymbol{n}^{T}\right\rangle\left(\boldsymbol{n}^{T}-\boldsymbol{n}^{S}\right)+\sum_{\ell=1}^{k-2}\left\langle\left(\boldsymbol{n}^{T}+\boldsymbol{n}^{S}\right)_{u_{i}}, \boldsymbol{n}_{\ell}^{S}\right\rangle \boldsymbol{n}_{\ell}^{S}-\sum_{j=1}^{s} h_{i}^{j}\left(\boldsymbol{n}^{T}, \boldsymbol{n}^{S}\right) \boldsymbol{X}_{u_{j}}$

(b) $\pi^{t} \circ \mathbb{L} \mathbb{G}\left(\boldsymbol{n}^{T}, \boldsymbol{n}^{S}\right)_{u_{i}}=-\sum_{j=1}^{s} h_{i}^{j}\left(\boldsymbol{n}^{T}, \boldsymbol{n}^{S}\right) \boldsymbol{X}_{u_{j}}$.

Here $\left(h_{i}^{j}\left(\boldsymbol{n}^{T}, \boldsymbol{n}^{S}\right)\right)=\left(h_{i k}\left(\boldsymbol{n}^{T}, \boldsymbol{n}^{S}\right)\right)\left(g^{k j}\right)$ and $\left(g^{k j}\right)=\left(g_{k j}\right)^{-1}$.

As a corollary of the above proposition, we have an explicit expression of the lightcone curvature in terms of the Riemannian metric and the lightcone second fundamental invariant.

Corollary 3.2 Under the same notations as in the above proposition, the lightcone LipschitzKilling curvature relative to $\left(\boldsymbol{n}^{T}, \boldsymbol{n}^{S}\right)$ is given by

$$
K_{\ell}\left(\boldsymbol{n}^{T}, \boldsymbol{n}^{S}\right)=\frac{\operatorname{det}\left(h_{i j}\left(\boldsymbol{n}^{T}, \boldsymbol{n}^{S}\right)\right)}{\operatorname{det}\left(g_{\alpha \beta}\right)} .
$$

Since $\left\langle-\left(\boldsymbol{n}^{T}+\boldsymbol{n}^{S}\right)(u), \boldsymbol{X}_{u_{j}}(u)\right\rangle=0$, we have $h_{i j}\left(\boldsymbol{n}^{T}, \boldsymbol{n}^{S}\right)(u)=\left\langle\boldsymbol{n}^{T}(u)+\boldsymbol{n}^{S}(u), \boldsymbol{X}_{u_{i} u_{j}}(u)\right\rangle$. Therefore the lightcone second fundamental invariant at a point $p_{0}=\boldsymbol{X}\left(u_{0}\right)$ depends only on the values $\boldsymbol{n}^{T}\left(u_{0}\right)+\boldsymbol{n}^{S}\left(u_{0}\right)$ and $\boldsymbol{X}_{u_{i} u_{j}}\left(u_{0}\right)$, respectively assumed by the vector fields $\boldsymbol{n}^{T}+\boldsymbol{n}^{S}$ and $\boldsymbol{X}_{u_{i} u_{j}}$ at the point $p_{0}$. Thus, the lightcone curvature also depends only on $\boldsymbol{n}^{T}\left(u_{0}\right)+\boldsymbol{n}^{S}\left(u_{0}\right)$, $\boldsymbol{X}_{u_{i}}\left(u_{0}\right)$ and $\boldsymbol{X}_{u_{i} u_{j}}\left(u_{0}\right)$, independent of the derivation of the vector fields $\boldsymbol{n}^{T}$ and $\boldsymbol{n}^{S}$. We write $K_{\ell}\left(\boldsymbol{n}_{0}^{T}, \boldsymbol{n}_{0}^{S}\right)\left(u_{0}\right)$ as the lightcone curvatures at $p_{0}=\boldsymbol{X}\left(u_{0}\right)$ with respect to $\left(\boldsymbol{n}_{0}^{T}, \boldsymbol{n}_{0}^{S}\right)=$ $\left(\boldsymbol{n}^{T}\left(u_{0}\right), \boldsymbol{n}^{S}\left(u_{0}\right)\right)$. We can also say that a point $p_{0}=\boldsymbol{X}\left(u_{0}\right)$ is $\left(\boldsymbol{n}_{0}^{T}, \boldsymbol{n}_{0}^{S}\right)$-umbilic because the lightcone $\left(\boldsymbol{n}^{T}, \boldsymbol{n}^{S}\right)$-shape operator at $p_{0}$ depends only on the normal vectors $\left(\boldsymbol{n}_{0}^{T}, \boldsymbol{n}_{0}^{S}\right)$. So we denote that $h_{i j}\left(\boldsymbol{n}^{T}, \boldsymbol{\xi}\right)\left(u_{0}\right)=h_{i j}\left(\boldsymbol{n}^{T}, \boldsymbol{n}^{S}\right)\left(u_{0}\right)$ and $K_{\ell}\left(\boldsymbol{n}^{T}, \boldsymbol{\xi}\right)\left(p_{0}\right)=K_{\ell}\left(\boldsymbol{n}_{0}^{T}, \boldsymbol{n}_{0}^{S}\right)\left(p_{0}\right)$, where $\boldsymbol{\xi}=$ $\boldsymbol{n}^{S}\left(u_{0}\right)$ for some local extension $\boldsymbol{n}^{T}(u)$ of $\boldsymbol{\xi}$. Analogously, we say that a point $p_{0}=\boldsymbol{X}\left(u_{0}\right)$ is an $\left(\boldsymbol{n}_{0}^{T}, \boldsymbol{n}_{0}^{S}\right)$-parabolic point of $\boldsymbol{X}: U \longrightarrow \mathbb{R}_{1}^{n+1}$ if $K_{\ell}\left(\boldsymbol{n}_{0}^{T}, \boldsymbol{n}_{0}^{S}\right)\left(u_{0}\right)=0$. And we say that a point $p_{0}=\boldsymbol{X}\left(u_{0}\right)$ is an $\left(\boldsymbol{n}_{0}^{T}, \boldsymbol{n}_{0}^{S}\right)$-flat point if it is an $\left(\boldsymbol{n}_{0}^{T}, \boldsymbol{n}_{0}^{S}\right)$-umbilic point and $K_{\ell}\left(\boldsymbol{n}_{0}^{T}, \boldsymbol{n}_{0}^{S}\right)\left(u_{0}\right)=0$.

On the other hand, the lightcone Gauss map $\widetilde{\mathbb{L} G}\left(\boldsymbol{n}^{T}, \boldsymbol{n}^{S}\right)$ with respect to $\left(\boldsymbol{n}^{T}, \boldsymbol{n}^{S}\right)$ also induces a linear mapping $d_{p} \widetilde{\mathbb{L} G}\left(\boldsymbol{n}^{T}, \boldsymbol{n}^{S}\right): T_{p} M \longrightarrow T_{p} \mathbb{R}_{1}^{n+1}$ under the identification of $U$ and $M$, where $p=\boldsymbol{X}(u)$. We have the following proposition. 
Proposition 3.3 Under the above notations, we have the following normalized lightcone Weingarten formula with respect to $\left(\boldsymbol{n}^{T}, \boldsymbol{n}^{S}\right)$ :

$$
\pi^{t} \circ \widetilde{\mathbb{L} \mathbb{G}}\left(\boldsymbol{n}^{T}, \boldsymbol{n}^{S}\right)_{u_{i}}(u)=-\sum_{j=1}^{s} \frac{1}{\ell_{0}(u)} h_{i}^{j}\left(\boldsymbol{n}^{T}, \boldsymbol{n}^{S}\right)(u) \boldsymbol{X}_{u_{j}}(u),
$$

where $\mathbb{L} \mathbb{G}\left(\boldsymbol{n}^{T}, \boldsymbol{n}^{S}\right)(u)=\left(\ell_{0}(u), \ell_{1}(u), \ldots, \ell_{n}(u)\right)$.

Proof. By definition, we have $\ell_{0} \widetilde{\mathbb{L} G}\left(\boldsymbol{n}^{T}, \boldsymbol{n}^{S}\right)=\mathbb{L} \mathbb{G}\left(\boldsymbol{n}^{T}, \boldsymbol{n}^{S}\right)$. It follows that $\ell_{0} \widetilde{\mathbb{L} G}\left(\boldsymbol{n}^{T}, \boldsymbol{n}^{S}\right)_{u_{i}}=$ $\mathbb{L} \mathbb{G}\left(\boldsymbol{n}^{T}, \boldsymbol{n}^{S}\right)_{u_{i}}-\ell_{0 u_{i}} \widetilde{\mathbb{L} \mathbb{G}}\left(\boldsymbol{n}^{T}, \boldsymbol{n}^{S}\right)$. Since $\widetilde{\mathbb{L} \mathbb{G}}\left(\boldsymbol{n}^{T}, \boldsymbol{n}^{S}\right)(u) \in N_{p}(M)$, we have

$$
\pi^{t} \circ \widetilde{\mathbb{L} \mathbb{G}}\left(\boldsymbol{n}^{T}, \boldsymbol{n}^{S}\right)_{u_{i}}=\frac{1}{\ell_{0}} \pi^{t} \circ \mathbb{L} \mathbb{G}\left(\boldsymbol{n}^{T}, \boldsymbol{n}^{S}\right)_{u_{i}} .
$$

By the lightcone Weingarten formula with respect to $\left(\boldsymbol{n}^{T}, \boldsymbol{n}^{S}\right)$ (Proposition 3.1), we have the desired formula.

We call the linear transformation $\widetilde{S}_{p}=-\pi^{t} \circ d_{p} \widetilde{\mathbb{L} G}\left(\boldsymbol{n}^{T}, \boldsymbol{n}^{S}\right)$ the normalized lightcone shape operator of $M$ at $p$ with respect to $\left(\boldsymbol{n}^{T}, \boldsymbol{n}^{S}\right)$. The eigenvalues $\left\{\widetilde{\kappa}_{i}\left(\boldsymbol{n}^{T}, \boldsymbol{n}^{S}\right)(p)\right\}_{i=1}^{s}$ of $\widetilde{S}_{p}$ are called the normalized lightcone principal curvatures. By the above proposition, we have $\widetilde{\kappa}_{i}\left(\boldsymbol{n}^{T}, \boldsymbol{n}^{S}\right)(p)=$ $\left(1 / \ell_{0}(u)\right) \kappa_{i}\left(\boldsymbol{n}^{T}, \boldsymbol{n}^{S}\right)(p)$. The normalized Lipschitz-Killing curvature of $M$ with respect to $\left(\boldsymbol{n}^{T}, \boldsymbol{n}^{S}\right)$ is defined to be $\widetilde{K}_{\ell}(u)=\operatorname{det} \widetilde{S}_{p}$. Then we have the following relation between the normalized lightcone Lipschitz-Killing curvature and the lightcone Lipschitz-Killing curvature:

$$
\widetilde{K}_{\ell}\left(\boldsymbol{n}^{T}, \boldsymbol{n}^{S}\right)(u)=\left(\frac{1}{\ell_{0}(u)}\right)^{s} K_{\ell}\left(\boldsymbol{n}^{T}, \boldsymbol{n}^{S}\right)(u)
$$

By definition, $p_{0}=\boldsymbol{X}\left(u_{0}\right)$ is the $\left(\boldsymbol{n}_{0}^{T}, \boldsymbol{n}_{0}^{S}\right)$-umbilical point if and only if $\widetilde{S}_{p_{0}}=\widetilde{\kappa}_{i}\left(\boldsymbol{n}^{T}, \boldsymbol{n}^{S}\right)\left(p_{0}\right) 1_{T_{p_{0}} M}$. We have the following proposition.

Proposition 3.4 Let $\boldsymbol{n}^{T}$ be a future directed timelike unit normal vector field along $M=$ $\boldsymbol{X}(U)$. Then the following conditions (1) and (2) are equivalent:

(1) There exists a spacelike unit normal vector field $\boldsymbol{n}^{S}$ along $M=\boldsymbol{X}(U)$ with $\left\langle\boldsymbol{n}^{T}, \boldsymbol{n}^{S}\right\rangle=0$ such that the normalized lightcone Gauss map $\widetilde{\mathbb{L} G}\left(\boldsymbol{n}^{T}, \boldsymbol{n}^{S}\right)$ of $M=\boldsymbol{X}(U)$ with respect to $\left(\boldsymbol{n}^{T}, \boldsymbol{n}^{S}\right)$ is constant.

(2) There exists $\boldsymbol{v} \in S_{+}^{n-1}$ and a real number c such that $M \subset H P(\boldsymbol{v}, c)$.

Suppose that the above condition holds. Then

(3) $M=\boldsymbol{X}(U)$ is totally $\left(\boldsymbol{n}^{T}, \boldsymbol{n}^{S}\right)$-flat.

Proof. Suppose that the condition (1) holds. We consider a function $F: U \longrightarrow \mathbb{R}$ defined by $F(u)=\langle\boldsymbol{X}(u), \boldsymbol{v}\rangle$. By definition, we have

$$
\frac{\partial F}{\partial u_{i}}(u)=\left\langle\boldsymbol{X}_{u_{i}}(u), \boldsymbol{v}\right\rangle=\left\langle\boldsymbol{X}_{u_{i}}(u), \widetilde{\mathbb{L} \mathbb{G}}\left(\boldsymbol{n}^{T}, \boldsymbol{n}^{S}\right)(u)\right\rangle
$$

for any $i=1, \ldots, s$. Therefore, $F(u)=\langle\boldsymbol{X}(u), \boldsymbol{v}\rangle=c$ is constant. It follows that $M \subset H P(\boldsymbol{v}, c)$ for $v \in S_{+}^{n-1}$.

Suppose that $M$ is a subset of a lightlike hyperplane $H(\boldsymbol{v}, c)$ for $\boldsymbol{v} \in S_{+}^{N-1}$. Since $M \subset$ $H P(\boldsymbol{v}, c)$, we have $T_{p} M \subset H(\boldsymbol{v}, 0)$. If $\left\langle\boldsymbol{n}^{T}(u), \boldsymbol{v}\right\rangle=0$, then $\boldsymbol{n}^{T}(u) \in H P(\boldsymbol{v}, 0)$. We remark that 
$H P(\boldsymbol{v}, 0)$ does not include timelike vectors. This is a contradiction. So we have $\left\langle\boldsymbol{n}^{T}(u), \boldsymbol{v}\right\rangle \neq 0$. We now define a normal vector field along $M=\boldsymbol{X}(U)$ by

$$
\boldsymbol{n}^{S}(u)=\frac{-1}{\left\langle\boldsymbol{n}^{T}(u), \boldsymbol{v}\right\rangle} \boldsymbol{v}-\boldsymbol{n}^{T}(u) .
$$

We can easily show that $\left\langle\boldsymbol{n}^{S}(u), \boldsymbol{n}^{S}(u)\right\rangle=1$ and $\left\langle\boldsymbol{n}^{S}(u), \boldsymbol{n}^{T}\right\rangle=0$. Therefore $\left(\boldsymbol{n}^{T}, \boldsymbol{n}^{S}\right)$ is a future directed orthonormal pair such that $\widetilde{\mathbb{L} G}\left(\boldsymbol{n}^{T}, \boldsymbol{n}^{S}\right)(u)=\boldsymbol{v}$.

On the other hand, by Proposition 3.3, if $\widetilde{\mathbb{L} G}\left(\boldsymbol{n}^{T}, \boldsymbol{n}^{S}\right)$ is constant, then $\left(h_{i}^{j}\left(\boldsymbol{n}^{T}, \boldsymbol{n}^{S}\right)(u)\right)=O$, so that $M=\boldsymbol{X}(U)$ is lightcone $\left(\boldsymbol{n}^{T}, \boldsymbol{n}^{S}\right)$-flat.

Suppose that $M=\boldsymbol{X}(U)$ is a codimension two spacelike submanifold in $\mathbb{R}_{1}^{n+1}$. In this case the pseudo-normal space $N_{p}(M)$ is a Lorenz plane, so that the lightcone is a set of two lightlike lines. Therefore, the directions $\boldsymbol{n}^{T} \pm \boldsymbol{n}^{S}$ are uniquely determined. Thus, the normalized Gauss map is independent of the choice of $\boldsymbol{n}^{T}$. We may write $\widetilde{\mathbb{L} G}=\widetilde{\mathbb{L} G}\left(\boldsymbol{n}^{T}, \boldsymbol{n}^{S}\right)$. The detailed geometric properties related to $\widetilde{\mathbb{L} G}$ were given in [11].

\section{Lightcone height functions}

In order to investigate the geometric meanings of the normalized lightcone Lipschitz-Killing curvature $\widetilde{K}_{\ell}\left(\boldsymbol{n}^{T}, \boldsymbol{n}^{S}\right)$ of $M=\boldsymbol{X}(U)$, we introduce a family of functions on $M=\boldsymbol{X}(U)$. We define the family of lightcone height functions $H: U \times S_{+}^{n-1} \longrightarrow \mathbb{R}$ on $M=\boldsymbol{X}(U)$ by $H(u, \boldsymbol{v})=$ $\langle\boldsymbol{X}(u), \boldsymbol{v}\rangle$. We denote the Hessian matrix of the lightcone height function $h_{\boldsymbol{v}_{0}}(u)=H\left(u, \boldsymbol{v}_{0}\right)$ at $u_{0}$ as Hess $\left(h_{v_{0}}\right)\left(u_{0}\right)$. The following proposition characterizes the lightlike parabolic points and lightlike flat points in terms of the family of lightcone height functions.

Proposition 4.1 Let $\boldsymbol{n}^{T}$ be a future directed timelike unit normal vector field along $M=\boldsymbol{X}(U)$ and $H: U \times S_{+}^{n-1} \longrightarrow \mathbb{R}$ the family of lightcone height functions on $M=\boldsymbol{X}(U)$. Then

(1) $\left(\partial H / \partial u_{i}\right)\left(u_{0}, \boldsymbol{v}_{0}\right)=0(i=1, \ldots, s)$ if and only if there exists a spacelike unit normal vector field $\boldsymbol{n}^{S}$ along $M=\boldsymbol{X}(U)$ with $\left\langle\boldsymbol{n}^{T}, \boldsymbol{n}^{S}\right\rangle=0$ such that $\boldsymbol{v}_{0}=\widetilde{\mathbb{L} G}\left(\boldsymbol{n}_{0}^{T}, \boldsymbol{n}_{0}^{S}\right)\left(u_{0}\right)$.

Suppose that $p_{0}=\boldsymbol{X}\left(u_{0}\right), \boldsymbol{v}_{0}=\widetilde{\mathbb{L} \mathbb{G}}\left(\boldsymbol{n}_{0}^{T}, \boldsymbol{n}_{0}^{S}\right)\left(u_{0}\right)$. Then

(2) $p_{0}$ is an $\left(\boldsymbol{n}_{0}^{T}, \boldsymbol{n}_{0}^{S}\right)$-parabolic point if and only if $\operatorname{det} \operatorname{Hess}\left(h_{\boldsymbol{v}_{0}}\right)\left(u_{0}\right)=0$,

(3) $p_{0}$ is a flat $\left(\boldsymbol{n}_{0}^{T}, \boldsymbol{n}_{0}^{S}\right)$-umbilic point if and only if $\operatorname{rank} \operatorname{Hess}\left(h_{\boldsymbol{v}_{0}}\right)\left(u_{0}\right)=0$.

Proof. (1) Since $\left(\partial H / \partial u_{i}\right)\left(u_{0}, \boldsymbol{v}_{0}\right)=\left\langle\boldsymbol{X}_{u_{i}}\left(u_{0}\right), \boldsymbol{v}_{0}\right),\left(\partial H / \partial u_{i}\right)\left(u_{0}, \boldsymbol{v}_{0}\right)=0(i=1, \ldots, s)$ if and only if $\boldsymbol{v}_{0} \in N_{p_{0}}(M)$ and $\boldsymbol{v}_{0} \in S_{+}^{n-1}$. By the same construction as in the proof of Proposition 3.4, we have a spacelike unit normal vector field $\boldsymbol{n}^{S}$ along $M=\boldsymbol{X}(U)$ with $\left\langle\boldsymbol{n}^{T}, \boldsymbol{n}^{S}\right\rangle=0$ such that $\boldsymbol{v}_{0}=\widetilde{\mathbb{L} G}\left(\boldsymbol{n}^{T}, \boldsymbol{n}^{S}\right)\left(u_{0}\right)=\widetilde{\mathbb{L} G}\left(\boldsymbol{n}_{0}^{T}, \boldsymbol{n}_{0}^{S}\right)\left(u_{0}\right)$. The converse also holds. For the proof of the assertions (2) and (3), as a consequence of Proposition 3.1, we have

$$
\begin{aligned}
\operatorname{Hess}\left(h_{\boldsymbol{v}_{0}}\right)\left(u_{0}\right) & =\left(\left\langle\boldsymbol{X}_{u_{i} u_{j}}\left(u_{0}\right), \widetilde{\mathbb{L} \mathbb{G}}\left(\boldsymbol{n}^{T}, \boldsymbol{n}^{S}\right)\left(u_{0}\right)\right\rangle\right)=\left(\frac{1}{\ell_{0}}\left\langle\boldsymbol{X}_{u_{i} u_{j}}\left(u_{0}\right), \boldsymbol{n}^{T}\left(u_{0}\right)+\boldsymbol{n}^{S}\left(u_{0}\right)\right\rangle\right) \\
& =\left(\frac{1}{\ell_{0}}\left\langle\boldsymbol{X}_{u_{i}}\left(u_{0}\right),\left(\boldsymbol{n}^{T}+\boldsymbol{n}^{S}\right)_{u_{j}}\left(u_{0}\right)\right\rangle\right) \\
& =\left(\frac{1}{\ell_{0}}\left\langle\boldsymbol{X}_{u_{i}}\left(u_{0}\right),-\sum_{k=1}^{s} h_{j}^{k}\left(\boldsymbol{n}^{T}, \boldsymbol{n}^{S}\right)\left(u_{0}\right) \boldsymbol{X}_{u_{k}}\left(u_{0}\right)\right\rangle\right)=\left(-\frac{1}{\ell_{0}} h_{i j}\left(\boldsymbol{n}^{T}, \boldsymbol{n}^{S}\right)\left(u_{0}\right)\right) .
\end{aligned}
$$


By definition, $K_{\ell}\left(\boldsymbol{n}^{T}, \boldsymbol{n}^{S}\right)\left(u_{0}\right)=0$ if and only if $\operatorname{det}\left(h_{i j}\left(\boldsymbol{n}^{T}, \boldsymbol{n}^{S}\right)\left(u_{0}\right)\right)=0$. The assertion (2) holds. Moreover, $p_{0}$ is a flat $\left(\boldsymbol{n}_{0}^{T}, \boldsymbol{n}_{0}^{S}\right)$-umbilical point if and only if $\left(h_{i j}\left(\boldsymbol{n}^{T}, \boldsymbol{n}^{S}\right)\left(u_{0}\right)\right)=O$. So we have the assertion (3).

We also define a family of functions $\widetilde{H}: U \times L C^{*} \longrightarrow \mathbb{R}$ by $\widetilde{H}(u, \boldsymbol{v})=\langle\boldsymbol{X}(u), \widetilde{\boldsymbol{v}}\rangle-v_{0}$, where $\boldsymbol{v}=\left(v_{0}, v_{1}, \ldots, v_{n}\right)$. We call $\widetilde{H}$ the family of extended lightcone height functions of $M=\boldsymbol{X}(U)$. Since $\partial \widetilde{H} / \partial u_{i}=\partial H / \partial u_{i}$ for $i=1, \ldots, s$ and $\operatorname{Hess}\left(\widetilde{h}_{\boldsymbol{v}}\right)=\operatorname{Hess}\left(h_{\widetilde{\boldsymbol{v}}}\right)$, we have the following proposition as a corollary of Proposition 4.3.

Proposition 4.2 Let $\widetilde{H}: U \times L C^{*} \longrightarrow \mathbb{R}$ be the extended lightcone height function of $M=$ $\boldsymbol{X}(U)$. Then

(1) $\widetilde{H}\left(u_{0}, \boldsymbol{v}_{0}\right)=\left(\partial \widetilde{H} / \partial u_{i}\right)\left(u_{0}, \boldsymbol{v}_{0}\right)=0(i=1, \ldots, s)$ if and only if there exists a spacelike unit normal vector field $\boldsymbol{n}^{S}$ along $M=\boldsymbol{X}(U)$ with $\left\langle\boldsymbol{n}^{T}, \boldsymbol{n}^{S}\right\rangle=0$ such that

$$
\boldsymbol{v}_{0}=\left\langle\boldsymbol{X}\left(u_{0}\right), \widetilde{\mathbb{L} \mathbb{G}}\left(\boldsymbol{n}_{0}^{T}, \boldsymbol{n}_{0}^{S}\right)\left(u_{0}\right)\right\rangle \widetilde{\mathbb{L} \mathbb{G}}\left(\boldsymbol{n}_{0}^{T}, \boldsymbol{n}_{0}^{S}\right)\left(u_{0}\right) .
$$

Suppose that $p_{0}=\boldsymbol{X}\left(u_{0}\right), \boldsymbol{v}_{0}=\left\langle\boldsymbol{X}\left(u_{0}\right), \widetilde{\mathbb{L} G}\left(\boldsymbol{n}_{0}^{T}, \boldsymbol{n}_{0}^{S}\right)\left(u_{0}\right)\right\rangle \widetilde{\mathbb{L} \mathbb{G}}\left(\boldsymbol{n}_{0}^{T}, \boldsymbol{n}_{0}^{S}\right)\left(u_{0}\right)$. Then

(2) $p_{0}$ is an $\left(\boldsymbol{n}_{0}^{T}, \boldsymbol{n}_{0}^{S}\right)$-parabolic point if and only if $\operatorname{det} \operatorname{Hess}\left(\widetilde{h}_{\boldsymbol{v}_{0}}\right)\left(u_{0}\right)=0$.

(3) $p_{0}$ is a flat $\left(\boldsymbol{n}_{0}^{T}, \boldsymbol{n}_{0}^{S}\right)$-umbilic point if and only if $\operatorname{rank} \operatorname{Hess}\left(\widetilde{h}_{\boldsymbol{v}_{0}}\right)\left(u_{0}\right)=0$.

Proof. It follows from Proposition 4.1, (1) that $\left(\partial \widetilde{H} / \partial u_{i}\right)\left(u_{0}, \boldsymbol{v}_{0}\right)=0(i=1, \ldots, s)$ if and only if there exists a spacelike unit normal vector field $\boldsymbol{n}^{S}$ along $M=\boldsymbol{X}(U)$ with $\left\langle\boldsymbol{n}^{T}, \boldsymbol{n}^{S}\right\rangle=0$ such that $\boldsymbol{v}_{0}=\widetilde{\mathbb{L} G}\left(\boldsymbol{n}_{0}^{T}, \boldsymbol{n}_{0}^{S}\right)\left(u_{0}\right)$. Moreover, the condition $\widetilde{H}\left(u_{0}, \boldsymbol{v}_{0}\right)=0$ is equivalent the condition that $v_{0}=\left\langle\boldsymbol{X}\left(u_{0}\right), \widetilde{\mathbb{L} G}\left(\boldsymbol{n}_{0}^{T}, \boldsymbol{n}_{0}^{S}\right)\left(u_{0}\right)\right\rangle$, where $\boldsymbol{v}_{0}=\left(v_{0}, v_{1}, \ldots, v_{n}\right)$. This means that

$$
\boldsymbol{v}_{0}=\left\langle\boldsymbol{X}\left(u_{0}\right), \widetilde{\mathbb{L} \mathbb{G}}\left(\boldsymbol{n}_{0}^{T}, \boldsymbol{n}_{0}^{S}\right)\left(u_{0}\right)\right\rangle \widetilde{\mathbb{L} \mathbb{G}}\left(\boldsymbol{n}_{0}^{T}, \boldsymbol{n}_{0}^{S}\right)\left(u_{0}\right) .
$$

The assertions (2) and (3) directly follows from the assertion (2) and (3) of Proposition 3.3.

\section{Codimension two spacelike canal submanifolds}

In this section we define a codimension two spacelike submanifold in Lorentz-Minkowski space which has analogous properties with the canal hypersurface of a submanifold in Euclidean space.

We now consider a spacelike embedding $\boldsymbol{X}: W \longrightarrow \mathbb{R}_{1}^{n+1}$ from an open subset $W \subset \mathbb{R}^{s}$. Moreover, we take an open subset $U \subset W$ such that $\bar{U} \subset W$ is compact, where $\bar{U}$ is the closure of $U$ in $\mathbb{R}^{s}$. For any future directed timelike unit normal vector field $\boldsymbol{n}^{T}$ along $M=\boldsymbol{X}(U)$, there exists a pseudo-orthonormal frame $\left\{\boldsymbol{n}^{T}, \boldsymbol{n}_{1}^{S}, \ldots, \boldsymbol{n}_{k-1}^{S}\right\}$ of $N(M)$, so that we have a frame field

$$
\left\{\boldsymbol{X}_{u_{1}}, \ldots, \boldsymbol{X}_{u_{s}}, \boldsymbol{n}^{T}, \boldsymbol{n}_{1}^{S}, \ldots, \boldsymbol{n}_{k-1}^{S}\right\}
$$

of $\mathbb{R}_{1}^{n+1}$ along $M=\boldsymbol{X}(U)$. We define an $S^{k-1}$-family of the spacelike unit normal vector field

$$
\boldsymbol{N}^{S}(u, \mu)=\sum_{j=1}^{k-1} \mu_{j} \boldsymbol{n}_{j}^{S}(u) \in N(M)_{1}\left[\boldsymbol{n}^{T}\right]
$$


along $M=\boldsymbol{X}(U)$ for $\mu=\left(\mu_{1}, \ldots, \mu_{k-1}\right) \in S^{k-2} \subset \mathbb{R}^{k-1}$. This gives a parametrization of the spacelike unit $(k-2)$-spherical normal bundle over $M=\boldsymbol{X}(U)$ with respect to $\boldsymbol{n}^{T}$. Under the canonical identification of $T_{p} \mathbb{R}_{1}^{n+1}$ with $\mathbb{R}_{1}^{n+1}$, we have $N_{1}(M)_{p} \subset \mathbb{R}_{1}^{n+1}$ for any $p=\boldsymbol{X}(u)$. We define a set

$$
C M_{2}\left(\boldsymbol{n}^{T} ; r\right)=\left\{\boldsymbol{X}(u)+r \boldsymbol{\xi} \mid \boldsymbol{\xi} \in N_{1}(M)_{p}\left[\boldsymbol{n}^{T}\right], p=\boldsymbol{X}(u), u \in U\right\} \subset \mathbb{R}_{1}^{n+1} .
$$

By an arbitrary chosen psuedo-orthonormal frame $\left\{\boldsymbol{X}_{u_{1}}, \ldots, \boldsymbol{X}_{u_{s}}, \boldsymbol{n}^{T}, \boldsymbol{n}_{1}^{S}, \ldots \boldsymbol{n}_{k-1}^{S}\right\}$, we have a mapping

$$
\overline{\boldsymbol{X}}\left(\boldsymbol{n}^{T}, \boldsymbol{N}^{S} ; r\right): U \times S^{k-2} \longrightarrow \mathbb{R}_{1}^{n+1}
$$

defined by

$$
\overline{\boldsymbol{X}}\left(\boldsymbol{n}^{T}, \boldsymbol{N}^{S} ; r\right)(u, \mu)=\boldsymbol{X}(u)+r \boldsymbol{N}^{S}(u, \mu) .
$$

We have the following proposition.

Proposition 5.1 For sufficiently small $r>0, \overline{\boldsymbol{X}}\left(\boldsymbol{n}^{T}, \boldsymbol{N}_{1}^{S} ; r\right)$ is a spacelike immersion.

Proof. We consider the local coordinate neighborhood of $S^{k-1}$ :

$$
U_{1}^{+}=\left\{\left(\mu_{1}, \ldots, \mu_{k-1}\right) \in S^{k-1} \mid \mu_{1}>0\right\} .
$$

Then we have $\mu_{1}=\sqrt{1-\sum_{j=2}^{k-1} \mu_{j}^{2}}$. For $i=1, \ldots, s, j=2, \ldots k-1$, we have the following calculation:

$$
\begin{aligned}
& \frac{\partial \overline{\boldsymbol{X}}\left(\boldsymbol{n}^{T}, \boldsymbol{N}^{S} ; r\right)}{\partial u_{i}}=\boldsymbol{X}_{u_{i}}(u)+r \sum_{j=1}^{k-1} \mu_{j} \boldsymbol{n}_{j, u_{i}}^{S}(u) \\
& \frac{\partial \overline{\boldsymbol{X}}\left(\boldsymbol{n}^{T}, \boldsymbol{N}^{S} ; r\right)}{\partial \mu_{j}}=r\left(\boldsymbol{n}_{j}^{S}(u)-\frac{\mu_{j}}{\mu_{1}} \boldsymbol{n}_{1}^{S}(u)\right) .
\end{aligned}
$$

Since $\left\{\boldsymbol{X}_{u_{i}}(u), r\left(\boldsymbol{n}_{j}^{S}(u)-\left(\mu_{j} / \mu_{1}\right) \boldsymbol{n}_{1}^{S}(u)\right) \mid i=1, \ldots, s, j=1, \ldots, k-1\right\}$ is linearly independent,

$$
\left\{\boldsymbol{X}_{u_{i}}(u)+r \sum_{j=1}^{k-1} \mu_{j} \boldsymbol{n}_{j, u_{i}}^{S}(u), r\left(\boldsymbol{n}_{j}^{S}(u)-\frac{\mu_{j}}{\mu_{1}} \boldsymbol{n}_{1}^{S}(u)\right) \mid i=1, \ldots, s, j=1, \ldots, k-1\right\}
$$

is linearly independent for sufficiently small $r>0$. Since $\boldsymbol{X}_{u_{i}}(u)$ is spacelike and $\bar{U}$ is compact, $\boldsymbol{X}_{u_{i}}(u)+r \sum_{j=1}^{k-1} \mu_{j} \boldsymbol{n}_{j, u_{i}}^{S}(u)$ is spacelike for sufficiently small $r>0$ for any $u \in U$ (even if $\sum_{j=1}^{k-1} \mu_{j} \boldsymbol{n}_{j, u_{i}}^{S}(u)$ is timelike). By definition, $\boldsymbol{n}_{j}^{S}(u)-\frac{\mu_{j}}{\mu_{1}} \boldsymbol{n}_{1}^{S}(u)$ is always spacelike, so that $\overline{\boldsymbol{X}}\left(\boldsymbol{n}^{T}, \boldsymbol{n}_{1}^{S}, \ldots, \boldsymbol{n}_{k-1}^{S} ; r\right)$ is a spacelike immersion for sufficiently small $r>0$.

For the other local coordinates of $S^{k-1}$, we can apply the similar calculation to the above case. This completes the proof.

We suppose that $\overline{\boldsymbol{X}}\left(\boldsymbol{n}^{T}, \boldsymbol{N}_{1}^{S} ; r\right)$ is a spacelike immersion. For any $\boldsymbol{\xi} \in N_{1}(M)_{p}\left[\boldsymbol{n}^{T}\right]$, there exists $\mu \in S^{k-1}$ such that $\boldsymbol{\xi}=\boldsymbol{N}^{S}(u, \mu)$, where $p=\boldsymbol{X}(u)$. It follows that we have

$$
\overline{\boldsymbol{X}}\left(\boldsymbol{n}^{T}, \boldsymbol{N}^{S} ; r\right)\left(U \times S^{k-2}\right)=C M_{2}\left(\boldsymbol{n}^{T} ; r\right) .
$$

Therefore, $C M_{2}\left(\boldsymbol{n}^{T} ; r\right)$ is a codimension two spacelike submanifold in $\mathbb{R}_{1}^{n+1}$. We call $C M_{2}\left(\boldsymbol{n}^{T} ; r\right)$ a codimension two spacelike canal submanifold of $M=\boldsymbol{X}(U)$ with respect to $\boldsymbol{n}^{T}$. If we consider $-\boldsymbol{N}^{S}$ instead of $\boldsymbol{N}^{S}$, we have $\overline{\boldsymbol{X}}\left(\boldsymbol{n}^{T},-\boldsymbol{N}^{S} ; r\right)\left(U \times S^{k-2}\right)=C M_{2}\left(\boldsymbol{n}^{T} ; r\right)$. 
Inspired by the results of Propositions 4.1 and 4.2, we define mappings

$$
\widetilde{\mathbb{L} \mathbb{G}}\left(\boldsymbol{n}^{T} ; r\right): C M_{2}\left(\boldsymbol{n}^{T} ; r\right) \longrightarrow S_{+}^{n-1}
$$

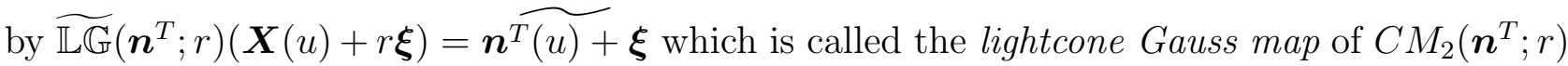
and

$$
\mathbb{L P}_{M}\left(\boldsymbol{n}^{T} ; r\right): C M_{2}\left(\boldsymbol{n}^{T} ; r\right) \longrightarrow L C^{*}
$$

by $\mathbb{L P}_{M}\left(\boldsymbol{n}^{T} ; r\right)(\boldsymbol{X}(u)+r \boldsymbol{\xi})=\left\langle\boldsymbol{X}(u),\left(\widetilde{\left.\boldsymbol{n}^{T(u)}+\boldsymbol{\xi}\right)}\right\rangle\left(\widetilde{\boldsymbol{n}^{T}(u)+\boldsymbol{\xi}}\right)\right.$. We call it the lightcone pedal hypersurface of $C M_{2}\left(\boldsymbol{n}^{T} ; r\right)$. Since the mappings $\overline{\boldsymbol{X}}\left(\boldsymbol{n}^{T}, \pm \boldsymbol{N}^{S} ; r\right)$ are parametrizations of $C M_{2}\left(\boldsymbol{n}^{T} ; r\right)$, we have the following parametrization of the above mappings:

$$
\begin{aligned}
& \widetilde{\mathbb{L} G}\left(\boldsymbol{n}^{T} ; r\right) \circ \overline{\boldsymbol{X}}\left(\boldsymbol{n}^{T}, \pm \boldsymbol{N}^{S} ; r\right)(u, \mu)=\boldsymbol{n}^{T}(u) \widetilde{\boldsymbol{N}^{S}}(u, \mu), \\
& \mathbb{L} \mathbb{P}_{M}\left(\boldsymbol{n}^{T} ; r\right) \circ \overline{\boldsymbol{X}}\left(\boldsymbol{n}^{T}, \pm \boldsymbol{N}^{S} ; r\right)(u, \mu)=\left\langle\boldsymbol{X}(u),\left(\boldsymbol{n}^{T}(u) \widetilde{\boldsymbol{N}^{S}}(u, \mu)\right)\right\rangle\left(\boldsymbol{n}^{T}(u) \pm \boldsymbol{N}^{S}(u, \mu)\right) .
\end{aligned}
$$

We respectively define the mappings

$$
\begin{aligned}
& \widetilde{\mathbb{L} G}\left(\boldsymbol{n}^{T}, \pm \boldsymbol{N}^{S}\right): U \times S^{k-2} \longrightarrow S_{+}^{n-1}, \\
& \mathbb{L} \mathbb{P}\left(\boldsymbol{n}^{T}, \pm \boldsymbol{N}^{S}\right): U \times S^{k-2} \longrightarrow L C^{*}
\end{aligned}
$$

by

$$
\begin{aligned}
& \widetilde{\mathbb{L} G}\left(\boldsymbol{n}^{T}, \pm \boldsymbol{N}^{S}\right)(u, \mu)=\boldsymbol{n}^{T}(u) \widetilde{\boldsymbol{N}^{S}}(u, \mu), \\
& \mathbb{L} \mathbb{P}\left(\boldsymbol{n}^{T}, \pm \boldsymbol{N}^{S}\right)(u, \mu)=\left\langle\boldsymbol{X}(u),\left(\boldsymbol{n}^{T}(u) \pm \boldsymbol{N}^{S}(u, \mu)\right)\right\rangle\left(\boldsymbol{n}^{T}(u) \pm \boldsymbol{N}^{S}(u, \mu)\right) .
\end{aligned}
$$

We now consider another frame field

$$
\left\{\boldsymbol{X}_{u_{1}}, \ldots, \boldsymbol{X}_{u_{s}}, \overline{\boldsymbol{n}}^{T}, \overline{\boldsymbol{n}}_{1}^{S}, \ldots, \overline{\boldsymbol{n}}_{k-1}^{S}\right\}
$$

of $\mathbb{R}_{1}^{n+1}$ along $M=\boldsymbol{X}(U)$ such that the ordered frames $\boldsymbol{n}^{T}, \boldsymbol{n}_{1}^{S}, \ldots, \boldsymbol{n}_{k-1}^{S}$ and $\overline{\boldsymbol{n}}^{T}, \overline{\boldsymbol{n}}_{1}^{S}, \ldots, \overline{\boldsymbol{n}}_{k-1}^{S}$ give the same orientation of the normal bundle $N(M)$. We have the following proposition.

Proposition 5.2 We have

$$
\widetilde{\mathbb{L} G}\left(\boldsymbol{n}^{T}, \pm \boldsymbol{N}^{S}\right)=\widetilde{\mathbb{L} G}\left(\overline{\boldsymbol{n}}^{T}, \pm \overline{\boldsymbol{N}} \bar{n}^{S}\right) \text { and } \mathbb{L} \mathbb{P}\left(\boldsymbol{n}^{T}, \pm \boldsymbol{N}^{S}\right)=\mathbb{L} \mathbb{P}\left(\overline{\boldsymbol{n}}^{T}, \pm \overline{\boldsymbol{N}} \overline{\boldsymbol{N}}^{S}\right)
$$

where $\overline{\boldsymbol{N}}^{S}(u, \mu)=\sum_{j=1}^{k-1} \mu_{j} \overline{\boldsymbol{n}}^{S}(u)$.

Proof. Since $\left\{\boldsymbol{n}^{T}, \boldsymbol{n}_{1}^{S}, \ldots, \boldsymbol{n}_{k-1}^{S}\right\}$ and $\left\{\overline{\boldsymbol{n}}^{T}, \overline{\boldsymbol{n}}_{1}^{S}, \ldots, \overline{\boldsymbol{n}}_{k-1}^{S}\right\}$ are the pseudo-orthonormal normal frame along $M=\boldsymbol{X}(U)$ and these give the same orientation of the normal bundle $N(M)$, there exists a smooth mapping $A: U \longrightarrow S O_{0}(1, k-1)$ such that $\boldsymbol{n}^{T}(u)=A(u) \overline{\boldsymbol{n}}^{T}(u), \boldsymbol{n}_{j}^{S}(u)=$ $A(u) \overline{\boldsymbol{n}}_{j}^{S}(u)$ for $j=1, \ldots k-1$. Then we have

$$
\boldsymbol{N}^{S}(u, \mu)=\sum_{j=1}^{k-1} \mu_{j} \boldsymbol{n}_{j}^{S}(u)=\sum_{j=1}^{k-1} \mu_{j} A(u) \overline{\boldsymbol{n}}_{j}^{S}(u)=A(u) \sum_{j=1}^{k-1} \mu_{j} \overline{\boldsymbol{n}}_{j}^{S}(u)=A(u) \overline{\boldsymbol{N}}_{j}^{S}(u, \mu) .
$$

Moreover, for any $\boldsymbol{\xi} \in N_{1}(M)_{p}\left[\overline{\boldsymbol{n}}^{T}\right]$, we have $A(u) \boldsymbol{\xi} \in N_{1}(M)_{p}\left[\boldsymbol{n}^{T}\right]$, where $p=\boldsymbol{X}(u)$. Therefore, we have the following diffeomorphism

$$
\Psi[A]: C M_{2}\left(\overline{\boldsymbol{n}}^{T} ; r\right) \longrightarrow C M_{2}\left(\boldsymbol{n}^{T} ; r\right)
$$


defined by $\Psi[A](\boldsymbol{X}(u)+r \boldsymbol{\xi})=\boldsymbol{X}(u)+r A(u) \boldsymbol{\xi}$. By definition, we have

$$
\Psi[A] \circ \overline{\boldsymbol{X}}\left(\overline{\boldsymbol{n}}^{T}, \pm \overline{\boldsymbol{N}}^{S} ; r\right)=\overline{\boldsymbol{X}}\left(\boldsymbol{n}^{T}, \pm \boldsymbol{N}^{S} ; r\right) \text { and } \widetilde{\mathbb{L} \mathbb{G}}\left(\boldsymbol{n}^{T} ; r\right) \circ \Psi[A]=\widetilde{\mathbb{L} \mathbb{G}}\left(\overline{\boldsymbol{n}}^{T} ; r\right) .
$$

It follows that

$$
\begin{aligned}
& \widetilde{\mathbb{L} G}\left(\overline{\boldsymbol{n}}^{T}, \pm \overline{\boldsymbol{N}} \overline{\boldsymbol{N}}^{S}\right)=\widetilde{\mathbb{L} \mathbb{G}}\left(\overline{\boldsymbol{n}}^{T} ; r\right) \circ \overline{\boldsymbol{X}}\left(\overline{\boldsymbol{n}}^{T}, \pm \overline{\boldsymbol{N}}{ }^{S} ; r\right) \\
& =\widetilde{\mathbb{L} \mathbb{G}}\left(\boldsymbol{n}^{T} ; r\right) \circ \Psi[A] \circ \overline{\boldsymbol{X}}\left(\overline{\boldsymbol{n}}^{T}, \pm \overline{\boldsymbol{N}} ; r\right)=\widetilde{\mathbb{L} \mathbb{G}}\left(\boldsymbol{n}^{T} ; r\right) \circ \overline{\boldsymbol{X}}\left(\boldsymbol{n}^{T}, \pm \boldsymbol{N}^{S} ; r\right)=\widetilde{\mathbb{L} \mathbb{G}}\left(\boldsymbol{n}^{T}, \pm \boldsymbol{N}^{S}\right) .
\end{aligned}
$$

By the same arguments as the above, we have $\mathbb{L} \mathbb{P}\left(\boldsymbol{n}^{T}, \pm \boldsymbol{N}^{S}\right)=\mathbb{L} \mathbb{P}\left(\overline{\boldsymbol{n}}^{T}, \pm \overline{\boldsymbol{N}}^{S}\right)$.

By the above proposition, we can denote that $\widetilde{\mathbb{L} \mathbb{G}^{ \pm}}=\widetilde{\mathbb{L} \mathbb{G}}\left(\boldsymbol{n}^{T}, \pm \boldsymbol{N}^{S}\right)$ and $\mathbb{L} \mathbb{P}^{ \pm}=\mathbb{L} \mathbb{P}\left(\boldsymbol{n}^{T}, \pm \boldsymbol{N}^{S}\right)$. We respectively call the suspended lightcone Gauss map and the suspended lightcone pedal hypersurface of $M=\boldsymbol{X}(U)$. We denote that $\widetilde{\mathbb{L G}}=\widetilde{\mathbb{L G}}+$ and $\mathbb{L P}=\mathbb{L} \mathbb{P}^{+}$.

\section{Contact viewpoint}

In this section we interpret the results of Propositions 4.1 and 4.2 from the view point of the contact with lightlike hyperplanes.

Firstly, we consider the relationship between the contact of submanifolds with foliations and the $\mathcal{R}^{+}$-classification of functions. Let $X_{i}(i=1,2)$ be submanifolds of $\mathbb{R}^{n}$ with $\operatorname{dim} X_{1}=$ $\operatorname{dim} X_{2}, g_{i}:\left(X_{i}, \bar{x}_{i}\right) \longrightarrow\left(\mathbb{R}^{n}, \bar{y}_{i}\right)$ be immersion germs and $f_{i}:\left(\mathbb{R}^{n}, \bar{y}_{i}\right) \longrightarrow(\mathbb{R}, 0)$ be submersion germs. For a submersion germ $f:\left(\mathbb{R}^{n}, 0\right) \longrightarrow(\mathbb{R}, 0)$, we denote that $\mathcal{F}_{f}$ be the regular foliation defined by $f$; i.e., $\mathcal{F}_{f}=\left\{f^{-1}(c) \mid c \in(\mathbb{R}, 0)\right\}$. We say that the contact of $X_{1}$ with the regular foliation $\mathcal{F}_{f_{1}}$ at $\bar{y}_{1}$ is of the same type as the contact of $X_{2}$ with the regular foliation $\mathcal{F}_{f_{2}}$ at $\bar{y}_{2}$ if there is a diffeomorphism germ $\Phi:\left(\mathbb{R}^{n}, \bar{y}_{1}\right) \longrightarrow\left(\mathbb{R}^{n}, \bar{y}_{2}\right)$ such that $\Phi\left(X_{1}\right)=$ $X_{2}$ and $\Phi\left(Y_{1}(c)\right)=Y_{2}(c)$, where $Y_{i}(c)=f_{i}^{-1}(c)$ for each $c \in(\mathbb{R}, 0)$. In this case we write $K\left(X_{1}, \mathcal{F}_{f_{1}} ; \bar{y}_{1}\right)=K\left(X_{2}, \mathcal{F}_{f_{2}} ; \bar{y}_{2}\right)$. It is clear that in the definition $\mathbb{R}^{n}$ could be replaced by any manifold. We apply the method of Goryunov [5] to the case for $\mathcal{R}^{+}$-equivalences among function germs, so that we have the following:

Proposition 6.1 ([5, Appendix $])$ Let $X_{i}(i=1,2)$ be submanifolds of $\mathbb{R}^{n}$ with $\operatorname{dim} X_{1}=$ $\operatorname{dim} X_{2}=n-1$ (i.e. hypersurface), $g_{i}:\left(X_{i}, \bar{x}_{i}\right) \longrightarrow\left(\mathbb{R}^{n}, \bar{y}_{i}\right)$ be immersion germs and $f_{i}$ : $\left(\mathbb{R}^{n}, \bar{y}_{i}\right) \longrightarrow(\mathbb{R}, 0)$ be submersion germs. Then $K\left(X_{1}, \mathcal{F}_{f_{1}} ; \bar{y}_{1}\right)=K\left(X_{2}, \mathcal{F}_{f_{2}} ; \bar{y}_{2}\right)$ if and only if $f_{1} \circ g_{1}$ and $f_{2} \circ g_{2}$ are $\mathcal{R}^{+}$-equivalent (i.e., there exists a diffeomorphism germ $\phi:\left(X_{1}, \bar{x}_{1}\right) \longrightarrow$ $\left(X_{2}, \bar{x}_{2}\right)$ such that $\left.\left(f_{2} \circ g_{2}\right) \circ \phi=f_{1} \circ g_{1}\right)$.

On the other hand, Golubitsky and Guillemin [4] have given an algebraic characterization for the $\mathcal{R}^{+}$-equivalence among function germs. We denote $C_{0}^{\infty}(X)$ is the set of function germs $(X, 0) \longrightarrow \mathbb{R}$. Let $J_{f}$ be the Jacobian ideal in $C_{0}^{\infty}(X)$ (i.e., $\left.J_{f}=\left\langle\partial f / \partial x_{1}, \ldots, \partial f / \partial x_{n}\right\rangle_{C_{0}^{\infty}(X)}\right)$. Let $\mathcal{R}_{k}(f)=C_{0}^{\infty}(X) / J_{f}^{k}$ and $\bar{f}$ be the image of $f$ in this local ring. We say that $f$ satisfies the Milnor condition if $\operatorname{dim}_{\mathbb{R}} \mathcal{R}_{1}(f)<\infty$.

Proposition 6.2 ([4, Proposition 4.1]) Let $f$ and $g$ be germs of functions at 0 in $X$ satisfying the Milnor condition with $d f(0)=d g(0)=0$. Then $f$ and $g$ are $\mathcal{R}^{+}$-equivalent if

(1) The rank and signature of the Hessians $\operatorname{Hess}(f)(0)$ and $\operatorname{Hess}(g)(0)$ are equal, and

(2) There is an isomorphism $\gamma: \mathcal{R}_{2}(f) \longrightarrow \mathcal{R}_{2}(g)$ such that $\gamma(\bar{f})=\bar{g}$. 
For $\boldsymbol{v}_{0}=\widetilde{\mathbb{L} G}\left(u_{0}, \mu_{0}\right)$, we consider a function $\mathfrak{h}_{\boldsymbol{v}_{0}}: \mathbb{R}_{1}^{n+1} \longrightarrow \mathbb{R}$ defined by $\mathfrak{h}_{\boldsymbol{v}_{0}}(\boldsymbol{x})=\left\langle\boldsymbol{x}, \boldsymbol{v}_{0}\right\rangle$. It is easy to show that $\mathfrak{h}_{\boldsymbol{v}_{0}}$ is a submersion. Moreover we have $\mathfrak{h}_{\boldsymbol{v}_{0}} \circ \boldsymbol{X}(u)=H\left(u, \boldsymbol{v}_{0}\right)$. By Proposition 3.2, we have

$$
\frac{\partial \mathfrak{h}_{\boldsymbol{v}_{0}} \circ \boldsymbol{X}}{\partial u_{i}}\left(u_{0}\right)=\frac{\partial H}{\partial u_{i}}\left(u_{0}, \boldsymbol{v}_{0}\right)=0 .
$$

for $i=1, \ldots, s$. This means that the lightlike hyperplane $\mathfrak{h}_{\boldsymbol{v}_{0}}{ }^{-1}(c)=H P\left(\boldsymbol{v}_{0}, c\right)$ is tangent to $M=\boldsymbol{X}(U)$ at $p_{0}=\boldsymbol{X}\left(u_{0}\right)$, where $c=\left\langle\boldsymbol{X}\left(u_{0}\right), \boldsymbol{v}_{0}\right\rangle$. In this case, we call $H P\left(\boldsymbol{v}_{0}, c\right)$ a tangent lightlike hyperplane with the pseudo-normal $\boldsymbol{v}_{0}$.

Since we have infinitely many lightlike directions in the pseudo-normal space, we have infinitely many tangent hyperplanes at each point $p_{0}=\boldsymbol{X}\left(u_{0}\right)$ depending on $\mu_{0} \in S^{k-2}$ (i.e., the direction of $\boldsymbol{N}^{S}\left(u_{0}, \mu_{0}\right)$. Let $\varepsilon$ be a sufficiently small positive real number. For any $t \in$ $I_{\varepsilon}=(c-\varepsilon, c+\varepsilon)$, we have a lightlike hyperplane $H P\left(\boldsymbol{v}_{0}, t\right)=\mathfrak{h}_{\boldsymbol{v}_{0}}^{-1}(t)$. In this case $\mathcal{F}_{\mathfrak{h} \boldsymbol{v}_{0}}$ is a family of parallel lightlike hyperplanes around $p=\boldsymbol{X}\left(u_{0}\right)$ such that $\mathfrak{h}_{\boldsymbol{v}_{0}}^{-1}(c)$ is the tangent lightlike hyperplane of $M$ at $p=\boldsymbol{X}\left(u_{0}\right)$ with respect to the pseudo-normal $\boldsymbol{v}_{0}$. Let $\boldsymbol{X}_{i}$ : $\left(U, u_{i}\right) \longrightarrow\left(\mathbb{R}_{1}^{n+1}, \boldsymbol{X}_{i}\left(u_{i}\right)\right)(i=1,2)$ be spacelike embedding germs from $U \subset \mathbb{R}^{3}$, then we have $h_{i, \boldsymbol{v}_{i}}(u)=\mathfrak{h}_{\boldsymbol{v}_{i}} \circ \boldsymbol{X}_{i}(u)$. Then we have the following proposition as a corollary of Propositions 6.1 and 6.2 .

Proposition 6.3 Let $\boldsymbol{X}_{i}:\left(U, u_{i}\right) \longrightarrow\left(\mathbb{R}_{1}^{n+1}, p_{i}\right)(i=1,2)$ be spacelike embedding germs such that $h_{i, \boldsymbol{v}_{i}}$ satisfy the Milnor condition, where $\boldsymbol{v}_{i}=\widetilde{\mathbb{L} G}\left(u_{i}, \mu_{i}\right)$ are pseudo-normals of the tangent lightlike hyperplanes of $\boldsymbol{X}_{i}(U)$ at $p_{i}=\boldsymbol{X}_{i}\left(u_{i}\right)$. Then the following conditions are equivalent:

(1) $K\left(\boldsymbol{X}_{1}(U), \mathcal{F}_{\mathfrak{h}_{1}} ; p_{1}\right)=K\left(\boldsymbol{X}_{2}(U), \mathcal{F}_{\mathfrak{h}_{2}} ; p_{2}\right)$.

(2) $h_{1, \boldsymbol{v}_{1}}$ and $h_{2, \boldsymbol{v}_{2}}$ are $\mathcal{R}^{+}$-equivalent.

(3) (a) The rank and signature of the $\operatorname{Hess}\left(h_{1, \boldsymbol{v}_{1}}\right)\left(u_{1}\right)$ and $\operatorname{Hess}\left(h_{2, \boldsymbol{v}_{2}}\right)\left(u_{2}\right)$ are equal,

(b) There is an isomorphism $\gamma: \mathcal{R}_{2}\left(h_{1, \boldsymbol{v}_{1}}\right) \longrightarrow \mathcal{R}_{2}\left(h_{2, \boldsymbol{v}_{2}}\right)$ such that $\gamma\left(\overline{h_{1, \boldsymbol{v}_{1}}}\right)=\overline{h_{2, \boldsymbol{v}_{2}}}$.

Secondary, we consider the theory of contact with a single submanifold due to Montaldi[16]. Let $X_{i}, Y_{i}(i=1,2)$ be submanifolds of $\mathbb{R}^{n}$ with $\operatorname{dim} X_{1}=\operatorname{dim} X_{2}$ and $\operatorname{dim} Y_{1}=\operatorname{dim} Y_{2}$. We say that the contact of $X_{1}$ and $Y_{1}$ at $y_{1}$ is the same type as the contact of $X_{2}$ and $Y_{2}$ at $y_{2}$ if there is a diffeomorphism germ $\Phi:\left(\mathbb{R}^{n}, y_{1}\right) \longrightarrow\left(\mathbb{R}^{n}, y_{2}\right)$ such that $\Phi\left(X_{1}\right)=X_{2}$ and $\Phi\left(Y_{1}\right)=Y_{2}$. In this case we write $K\left(X_{1}, Y_{1} ; y_{1}\right)=K\left(X_{2}, Y_{2} ; y_{2}\right)$. It is clear that in the definition $\mathbb{R}^{n}$ could be replaced by any manifold. In his paper [16] Montaldi gives a characterization of the notion of contact by using the terminology of singularity theory.

Theorem 6.4 Let $X_{i}, Y_{i}(i=1,2)$ be submanifolds of $\mathbb{R}^{n}$ with $\operatorname{dim} X_{1}=\operatorname{dim} X_{2}$ and $\operatorname{dim} Y_{1}=$ $\operatorname{dim} Y_{2}$. Let $g_{i}:\left(X_{i}, x_{i}\right) \longrightarrow\left(\mathbb{R}^{n}, y_{i}\right)$ be immersion germs and $f_{i}:\left(\mathbb{R}^{n}, y_{i}\right) \longrightarrow\left(\mathbb{R}^{p}, 0\right)$ be submersion germs with $\left(Y_{i}, y_{i}\right)=\left(f_{i}^{-1}(0), y_{i}\right)$. Then $K\left(X_{1}, Y_{1} ; y_{1}\right)=K\left(X_{2}, Y_{2} ; y_{2}\right)$ if and only if $f_{1} \circ g_{1}$ and $f_{2} \circ g_{2}$ are $\mathcal{K}$-equivalent (i.e., there exist a diffeomorphism germ $\phi:\left(X_{1}, \bar{x}_{1}\right) \longrightarrow$ $\left(X_{2}, \bar{x}_{2}\right)$ and a non-zero function germ $\lambda:\left(X_{1}, \bar{x}_{1}\right) \longrightarrow \mathbb{R}$ such that $\left.\lambda \cdot\left(f_{2} \circ g_{2}\right) \circ \phi=f_{1} \circ g_{1}\right)$.

We now consider a function $\widetilde{\mathfrak{h}}_{\boldsymbol{v}}: \mathbb{R}_{1}^{n+1} \longrightarrow \mathbb{R}$ defined by $\widetilde{\mathfrak{h}}_{\boldsymbol{v}}(\boldsymbol{u})=\langle\boldsymbol{u}, \widetilde{\boldsymbol{v}}\rangle-v_{0}$, where $\boldsymbol{v}=\left(v_{0}, v_{1}, \ldots, v_{n}\right)$. For any $\boldsymbol{v}_{*} \in L C^{*}$ and $p_{0}=\boldsymbol{X}\left(u_{0}\right)$, we have a lightlike hyperplane $\mathfrak{h}_{\boldsymbol{v}_{*}}^{-1}(0)=$ $H P\left(\boldsymbol{v}_{*}, v_{0}\right)$. Moreover, we consider the lightlike vector $\boldsymbol{v}_{*}=\mathbb{L} \mathbb{P}\left(u_{0}, \mu_{0}\right)$, then we have

$$
\widetilde{\mathfrak{h}}_{\boldsymbol{v}_{*}} \circ \boldsymbol{X}\left(u_{0}\right)=\widetilde{H}\left(u_{0}, \mathbb{L} \mathbb{P}\left(u_{0}, \mu_{0}\right)\right)=0 .
$$


By Proposition 4.2, we also have relations that

$$
\frac{\partial \widetilde{\mathfrak{h}}_{\boldsymbol{v}_{*}} \circ \boldsymbol{X}}{\partial u_{i}}\left(u_{0}\right)=\frac{\partial \widetilde{H}}{\partial u_{i}}\left(u_{0}, \mathbb{L} \mathbb{P}\left(u_{0}, \mu_{0}\right)\right)=0 .
$$

for $i=1, \ldots, s$. This means that the lightlike hyperplane $\widetilde{\mathfrak{h}}_{\boldsymbol{v}_{*}}^{-1}(0)=H P\left(\boldsymbol{v}_{*}, v_{0}\right)$ is tangent to $M=\boldsymbol{X}(U)$ at $p_{0}=\boldsymbol{X}\left(u_{0}\right)$. The lightlike hypersurface $H P\left(\boldsymbol{v}_{*}, v_{0}\right)$ is a tangent lightlike hyperplane of $M=\boldsymbol{X}(U)$ at $p_{0}=\boldsymbol{X}\left(u_{0}\right)$, which we write $\operatorname{THP}\left(M, \mathbb{L} \mathbb{P}\left(u_{0}, \mu_{0}\right)\right)$. Then we have the following simple lemma.

Lemma 6.5 Let $\boldsymbol{X}: U \longrightarrow \mathbb{R}_{1}^{n+1}$ be a spacelike submanifold with dimension s. Consider two points $\left(u_{1}, \mu_{1}\right),\left(u_{2}, \mu_{2}\right) \in U \times S^{k-2}$. Then $\mathbb{L} \mathbb{P}\left(u_{1}, \mu_{1}\right)=\mathbb{L} \mathbb{P}\left(u_{2}, \mu_{2}\right)$ if and only if

$$
\operatorname{THP}\left(M,\left(u_{1}, \mu_{1}\right)\right)=\operatorname{THP}\left(M,\left(u_{2}, \mu_{2}\right)\right) .
$$

Eventually, we have tools for the study of the contact between spacelike hypersurfaces and lightlike hyperplane. Since we have $\widetilde{h}_{\boldsymbol{v}}(u)=\widetilde{\mathfrak{h}}_{\boldsymbol{v}} \circ \boldsymbol{X}(u)$, we have the following proposition as a corollary of Theorem 6.4.

Proposition 6.6 Let $\boldsymbol{X}_{i}:\left(U, u_{i}\right) \longrightarrow\left(\mathbb{R}_{1}^{n+1}, p_{i}\right)(i=1,2)$ be spacelike embedding germs and $\boldsymbol{v}_{i}=\mathbb{L} \mathbb{P}\left(u_{i}, \mu_{i}\right)$. We write that $M_{i}=\boldsymbol{X}_{i}(U)$. Then the following conditions are equivalent:

(1) $K\left(M_{1}, \operatorname{THP}\left(M,\left(u_{1}, \mu_{1}\right)\right) ; p_{1}\right)=K\left(M_{2}, \operatorname{THP}\left(M,\left(u_{2}, \mu_{2}\right)\right) ; p_{2}\right)$,

(2) $\widetilde{h}_{1, \boldsymbol{v}_{1}}$ and $\widetilde{h}_{2, \boldsymbol{v}_{2}}$ are $\mathcal{K}$-equivalent.

\section{The view point from Lagrangian singularity theory}

In order to apply the theory of Lagrangian singularities to our situation, we give a brief review on the theory of Lagrangian singularities due to [1]. We consider the cotangent bundle $\pi: T^{*} \mathbb{R}^{n-1} \longrightarrow \mathbb{R}^{n-1}$ over $\mathbb{R}^{n-1}$. Let $(q, x)=\left(x_{1}, \ldots, x_{n-1}, p_{1}, \ldots, p_{n-1}\right)$ be the canonical coordinate on $T^{*} \mathbb{R}^{n-1}$. Then the canonical symplectic structure on $T^{*} \mathbb{R}^{n-1}$ is given by the canonical two form $\omega=\sum_{i=1}^{n-1} d p_{i} \wedge d x_{i}$. Let $i: L \longrightarrow T^{*} \mathbb{R}^{n-1}$ be an immersion. We say that $i(L)$ is a Lagrangian submanifold if $\operatorname{dim} L=n-1$ and $i^{*} \omega=0$. In this case the critical value of $\pi \circ i$ is called the caustic of $i: L \longrightarrow T^{*} \mathbb{R}^{n-1}$ and it is denoted by $C_{L}$. The main result in the theory of Lagrangian singularities is to describe Lagrangian immersion germs by using families of function germs. Let $F:\left(\mathbb{R}^{k} \times \mathbb{R}^{n-1},(\mathbf{0}, \mathbf{0})\right) \longrightarrow(\mathbb{R}, 0)$ be an $n+1$-parameter unfolding of function germs. We call

$$
C(F)=\left\{(q, x) \in\left(\mathbb{R}^{k} \times \mathbb{R}^{n-1},(\mathbf{0}, \mathbf{0})\right) \mid \frac{\partial F}{\partial q_{1}}(q, x)=\cdots=\frac{\partial F}{\partial q_{k}}(q, x)=0\right\},
$$

the catastrophe set of $F$ and

$$
B_{F}=\left\{x \in\left(\mathbb{R}^{n-1}, 0\right) \mid \text { there exist }(q, x) \in C(F) \text { such that } \operatorname{rank}\left(\frac{\partial^{2} F}{\partial q_{i} \partial j_{j}}(q, x)\right)<k\right\}
$$

the bifurcation set of $F$. Let $\pi_{n-1}:\left(\mathbb{R}^{k} \times \mathbb{R}^{n-1}, 0\right) \longrightarrow\left(\mathbb{R}^{n-1}, 0\right)$ be the canonical projection, then we can easily show that the bifurcation set of $F$ is the critical value set of $\pi_{n-1} \mid C(F)$. We say that $F$ is a Morse family of functions if the map germ

$$
\Delta F=\left(\frac{\partial F}{\partial q_{1}}, \ldots, \frac{\partial F}{\partial q_{k}}\right):\left(\mathbb{R}^{k} \times \mathbb{R}^{n-1}, 0\right) \longrightarrow\left(\mathbb{R}^{k}, 0\right)
$$


is non-singular, where $(q, x)=\left(q_{1}, \ldots, q_{k}, x_{1}, \ldots, x_{n-1}\right) \in\left(\mathbb{R}^{k} \times \mathbb{R}^{n-1}, 0\right)$. In this case we have a smooth submanifold germ $C(F) \subset\left(\mathbb{R}^{k} \times \mathbb{R}^{n-1}, 0\right)$ and a map germ $L_{F}:(C(F), 0) \longrightarrow T^{*} \mathbb{R}^{n-1}$ defined by

$$
L_{F}(q, x)=\left(x, \frac{\partial F}{\partial x_{1}}(q, x), \ldots, \frac{\partial F}{\partial x_{n-1}}(q, x)\right) .
$$

We can show that $L_{F}(C(F))$ is a Lagrangian submanifold germ. Then we have the following fundamental theorem ([1], page 300).

Proposition 7.1 All Lagrangian submanifold germs in $T^{*} \mathbb{R}^{n-1}$ are constructed by the above method.

Under the above notation, we call $F$ a generating family of $L_{F}(C(F))$.

We define an equivalence relation among Lagrangian submanifold germs. Let $i:(L, p) \subset$ $\left(T^{*} \mathbb{R}^{n-1}, p\right)$ and $i^{\prime}:\left(L^{\prime}, p^{\prime}\right) \subset\left(T^{*} \mathbb{R}^{n-1}, p^{\prime}\right)$ be Lagrangian submanifold germs. Then we say that $i$ and $i^{\prime}$ are Lagrangian equivalent if there exist a symplectic diffeomorphism germ $\tau$ : $\left(T^{*} \mathbb{R}^{n-1}, p\right) \longrightarrow\left(T^{*} \mathbb{R}^{n-1}, p^{\prime}\right)$ and a diffeomorphism germ $\bar{\tau}:\left(\mathbb{R}^{n-1}, \pi(p)\right) \longrightarrow\left(\mathbb{R}^{n-1}, \pi\left(p^{\prime}\right)\right)$ such that $\tau(L)=L^{\prime}$ and $\pi \circ \tau=\bar{\tau} \circ \pi$, where $\pi:\left(T^{*} \mathbb{R}^{n-1}, p\right) \longrightarrow\left(\mathbb{R}^{n-1}, \pi(p)\right)$ is the canonical projection. In this case the caustic $C_{L}$ is diffeomorphic to the caustic $C_{L^{\prime}}$ by the diffeomorphism germ $\bar{\tau}$. We can define the notion of Lagrangian stability for Lagrangian submanifold germs, but we omit the detail here (cf., [1]). We can interpret the Lagrangian equivalence by using the notion of generating families. We denote $\mathcal{E}_{m}$ the local ring of function germs $\left(\mathbb{R}^{m}, 0\right) \longrightarrow \mathbb{R}$ with the unique maximal ideal $\mathfrak{M}_{m}=\left\{h \in \mathcal{E}_{m} \mid h(0)=0\right\}$. Let $F, G:\left(\mathbb{R}^{k} \times \mathbb{R}^{n-1}, 0\right) \longrightarrow(\mathbb{R}, 0)$ be function germs. We say that $F$ and $G$ are $P-\mathcal{R}^{+}$-equivalent if there exists a diffeomorphism germ $\Phi:\left(\mathbb{R}^{k} \times \mathbb{R}^{n-1}, 0\right) \longrightarrow\left(\mathbb{R}^{k} \times \mathbb{R}^{n-1}, 0\right)$ of the form $\Phi(q, x)=\left(\Phi_{1}(q, x), \phi(x)\right)$ and a function germ $h:\left(\mathbb{R}^{n-1}, 0\right) \longrightarrow \mathbb{R}$ such that $G(q, x)=F(\Phi(q, x))+h(x)$. For any $F_{1} \in \mathfrak{M}_{k+n+1}$ and $F_{2} \in \mathfrak{M}_{k^{\prime}+n+1}, F_{1}, F_{2}$ are said to be stably $P$ - $\mathcal{R}^{+}$-equivalent if they become $P$ - $\mathcal{R}^{+}$-equivalent after the addition to the arguments to $q_{i}$ of new arguments $q_{i}^{\prime}$ and to the functions $F_{i}$ of non-degenerate quadratic forms $Q_{i}$ in the new arguments (i.e., $F_{1}+Q_{1}$ and $F_{2}+Q_{2}$ are $P$ $\mathcal{R}^{+}$-equivalent). Let $F:\left(\mathbb{R}^{k} \times \mathbb{R}^{n-1}, 0\right) \longrightarrow(\mathbb{R}, 0)$ be a function germ. We say that $F$ is an infinitesimally $\mathcal{R}^{+}$-versal deformation of $f=\left.F\right|_{\mathbb{R}^{k} \times\{0\}}$ if

$$
\mathcal{E}_{k}=J_{f}+\left\langle\frac{\partial F}{\partial x_{1}}\left|\mathbb{R}^{k} \times\{0\}, \ldots, \frac{\partial F}{\partial x_{n-1}}\right| \mathbb{R}^{k} \times\{0\}\right\rangle_{\mathbb{R}}+\langle 1\rangle_{\mathbb{R}},
$$

where

$$
J_{f}=\left\langle\frac{\partial f}{\partial q_{1}}, \ldots, \frac{\partial f}{\partial q_{k}}\right\rangle_{\mathcal{E}_{k}} .
$$

We have the following fundamental theorem (cf., [1], page 304 and 325).

Theorem 7.2 Let $F_{1} \in \mathfrak{M}_{k+n-1}$ and $F_{2} \in \mathfrak{M}_{k^{\prime}+n-1}$ be Morse families of functions. Then we have the following:

(1) $\left.L_{F_{1}}\left(C F_{1}\right)\right)$ and $L_{F_{2}}\left(C\left(F_{2}\right)\right)$ are Lagrangian equivalent if and only if $F_{1}, F_{2}$ are stably $P-\mathcal{R}^{+}$equivalent.

(2) $L_{F}(C(F))$ is Lagrangian stable if and only if $F$ is an infinitesimally $\mathcal{R}^{+}$-versal deformation of $F \mid \mathbb{R}^{k} \times\{0\}$.

The following proposition describes the well-known relationship between bifurcation sets and equivalence among unfoldings of function germs: 
Proposition 7.3 Let $F, G:\left(\mathbb{R}^{k} \times \mathbb{R}^{n-1}, 0\right) \longrightarrow(\mathbb{R}, 0)$ be function germs. If $F$ and $G$ are $P-\mathcal{R}^{+}$-equivalent then there exist a diffeomorphism germ $\phi:\left(\mathbb{R}^{n-1}, 0\right) \longrightarrow\left(\mathbb{R}^{n-1}, 0\right)$ such that $\phi\left(B_{F}\right)=B_{G}$.

We now apply the above theory of Lagrangian singularities to our situation. Firstly, we have the following proposition.

Proposition 7.4 The lightcone height function $H: U \times S_{+}^{n-1} \longrightarrow \mathbb{R}$ of $M=\boldsymbol{X}(U)$ is a Morse family of functions.

Proof. Let $\boldsymbol{X}=\left(X_{0}, \ldots, X_{n}\right)$ and $\boldsymbol{v}=\left(v_{0}, \ldots, v_{n}\right) \in S_{+}^{n-1}$, without loss of the generality, we assume that $v_{n}>0$, then we may take a local coordinate $\left(v_{1}, \ldots, v_{n-1}\right)$ on $S_{+}^{n-1}$ and $v_{n}=\sqrt{1-v_{1}^{2}-\cdots-v_{n-1}^{2}}$. Let $\Delta H=\left(H_{u_{1}}, \ldots, H_{u_{s}}\right)$, we now prove a map $\Delta H$ is submersive at $\left(u_{0}, \boldsymbol{v}_{0}\right) \in C(H)$. Its Jacobian matrix $J \Delta H$ is

$$
J \Delta H=\left(\left(H_{u_{i} u_{j}}\right)_{i, j=1, \ldots, s} \mid\left(H_{u_{i} v_{j}}\right)_{i=1, \ldots, s, j=1, \ldots, n-1}\right) .
$$

It is sufficient show that the rank of a matrix $B(u, \boldsymbol{v}):=\left(H_{u_{i} v_{j}}(u, \boldsymbol{v})\right)_{i j}$ is $s$. By calculation, components of $B(u, \boldsymbol{v})$ are

$$
H_{u_{i} v_{j}}(u, \boldsymbol{v})=\left(X_{j}\right)_{u_{i}}(u)-\frac{v_{j}}{v_{n}}\left(X_{n}\right)_{u_{i}}(u)
$$

Let $\mathbf{e}_{0}=(1,0, \ldots, 0)$ be a timelike vector, then $\mathbf{e}_{0}, \boldsymbol{v}, \boldsymbol{X}_{u_{1}}(u), \ldots, \boldsymbol{X}_{u_{s}}(u)$ are linearly independent at $\left(u_{0}, \boldsymbol{v}_{0}\right)$. This means that the rank of the matrix

$$
\mathrm{C}=\left(\begin{array}{cccc}
1 & 0 & \cdots & 0 \\
1 & v_{1} & \cdots & v_{n} \\
\left(X_{0}\right)_{u_{1}} & \left(X_{1}\right)_{u_{1}} & \cdots & \left(X_{n}\right)_{u_{1}} \\
\vdots & \vdots & \ddots & \vdots \\
\left(X_{0}\right)_{u_{s}} & \left(X_{1}\right)_{u_{s}} & \cdots & \left(X_{n}\right)_{u_{s}}
\end{array}\right)
$$

is $s+2$ at $\left(u_{0}, \boldsymbol{v}_{0}\right)$. We now show that $\operatorname{rank} C=\operatorname{rank} B+2$. We subtract the second raw multiplied by $\left(X_{n}\right)_{u_{i}} / v_{n}$ from the $(2+i)$-th raw for $i=1, \ldots, s$. And we also subtract the first law multiplied by $(2+i, 1)$ component from the $(2+i)$-th raw for $i=1, \ldots, s$. Then we have

$$
\mathrm{C}^{\prime}=\left(\begin{array}{c|c|c}
1 & 0 \cdots 0 & 0 \\
\hline 1 & v_{1} \cdots v_{n-1} & v_{n} \\
\hline 0 & & 0 \\
\vdots & B(u, \boldsymbol{v}) & \vdots \\
0 & & 0
\end{array}\right)
$$

Therefore $\operatorname{rank} B\left(u_{0}, \boldsymbol{v}_{0}\right)=s$, this completes the proof.

Corollary 7.5 Under the above notations, $L_{H}(C(H))$ is a Lagrangian submanifold in $T^{*}\left(S^{n-1}\right)$ such that the lightcone height function $H: U \times S_{+}^{n-1} \longrightarrow \mathbb{R}$ is a generating family of $L_{H}(C(H))$ at least locally. 
By Proposition 4.1 and the arguments in $\S 5$, we have

$$
C(H)=\left\{(u, \widetilde{\mathbb{L} G}(u, \mu)) \mid(u, \mu) \in U \times S^{k-2}\right\}
$$

and $\pi \circ L_{H}=\widetilde{\mathbb{L} G}$, where $\pi: T^{*} S_{+}^{n-1} \longrightarrow S_{+}^{n-1}$ is the projection of the cotangent bundle.

Theorem 7.6 Let $\boldsymbol{X}_{i}:\left(U, \bar{u}_{i}\right) \longrightarrow\left(\mathbb{R}_{1}^{n+1}, p_{i}\right)$ be embedding germs of codimension $k(i=1,2)$. Suppose that the Lagrangian lift germs $L_{H_{i}}:\left(C\left(H_{i}\right),\left(\bar{u}_{i}, \boldsymbol{v}_{i}\right)\right) \longrightarrow\left(T^{*} S_{+}^{n-1}, \bar{z}_{i}\right)$ of the suspended lightcone Gauss map germs $\widetilde{\mathbb{L G}}_{i}$ are Lagrangian stable, where $\boldsymbol{v}_{i}=\widetilde{\mathbb{L}}_{i}\left(\bar{u}_{i}, \bar{\mu}_{1}\right)$. We write $M_{i}=\boldsymbol{X}_{i}(U)$ and $\mathcal{F}_{\mathfrak{h}_{i}}$ is the family of parallel lightlike hyperplane around $p_{i}=\boldsymbol{X}\left(\bar{u}_{i}\right)$. Then the following conditions are equivalent:

(1) $K\left(M_{1}, \mathcal{F}_{\mathfrak{h}_{\boldsymbol{v}_{1}}} ; p_{1}\right)=K\left(M_{2}, \mathcal{F}_{\mathfrak{h} \boldsymbol{v}_{2}} ; p_{2}\right)$.

(2) $h_{1, \boldsymbol{v}_{1}}$ and $h_{2, \boldsymbol{v}_{2}}$ are $\mathcal{R}^{+}$-equivalent.

(3) $H_{1}$ and $H_{2}$ are $P-\mathcal{R}^{+}$-equivalent.

(4) $L_{H_{1}}\left(C\left(H_{1}\right)\right)$ and $L_{H_{2}}\left(C\left(H_{2}\right)\right)$ are Lagrangian equivalent.

(5) (a) The rank and signature of the $\operatorname{Hess}\left(h_{1, \boldsymbol{v}_{1}}\right)\left(\bar{u}_{1}\right)$ and $\operatorname{Hess}\left(h_{2, \boldsymbol{v}_{2}}\right)\left(\bar{u}_{2}\right)$ are equal,

(b) There is an isomorphism $\gamma: \mathcal{R}_{2}\left(h_{1, \boldsymbol{v}_{1}}\right) \longrightarrow \mathcal{R}_{2}\left(h_{2, \boldsymbol{v}_{2}}\right)$ such that $\gamma\left(\overline{h_{1, \boldsymbol{v}_{1}}}\right)=\overline{h_{2, \boldsymbol{v}_{2}}}$.

Proof. We remark that if $L_{H_{i}}\left(C\left(H_{i}\right)\right)$ is Lagrangian stable, then Theorem 7.2, (2) implies that $h_{i, \boldsymbol{v}_{i}}$ satisfies the Milnor condition. Therefore, by Proposition 6.3, the conditions (1), (2) and (5) are equivalent. By the uniqueness of $\mathcal{R}^{+}$-versal deformation, the condition (2) implies the condition (3). By definition, the condition (3) implies the condition (2). By Theorem 7.2, (1), the conditions (3) and (4) are equivalent. This completes the proof.

In the above proof, we only need the assumption of the Lagrangian stability for the proof that the condition (2) implies the condition (3).

\section{The view point from Legendrian singularity theory}

In order to apply the theory of Legendrian singularities to our situation, we give a quick review on the Legendrian singularity theory mainly due to Arnol'd-Zakalyukin [1]. Let $\pi$ : $P T^{*}(M) \longrightarrow M$ be the projective cotangent bundle over an $n$-dimensional manifold $M$. This fibration can be considered as a Legendrian fibration with the canonical contact structure $K$ on $P T^{*}(M)$. We now review geometric properties of this space. Consider the tangent bundle $\tau: T P T^{*}(M) \rightarrow P T^{*}(M)$ and the differential map $d \pi: T P T^{*}(M) \rightarrow N$ of $\pi$. For any $X \in T P T^{*}(M)$, there exists an element $\alpha \in T^{*}(M)$ such that $\tau(X)=[\alpha]$. For an element $V \in T_{x}(M)$, the property $\alpha(V)=\mathbf{0}$ does not depend on the choice of representative of the class $[\alpha]$. Thus we can define the canonical contact structure on $\operatorname{PT}^{*}(M)$ by

$$
K=\left\{X \in T P T^{*}(M) \mid \tau(X)(d \pi(X))=0\right\}
$$

For a local coordinate neighborhood $\left(U,\left(x_{1}, \ldots, x_{n}\right)\right)$ on $M$, we have a trivialization $P T^{*}(U) \cong$ $U \times P\left(\mathbb{R}^{n-1}\right)^{*}$ and we call $\left(\left(x_{1}, \ldots, x_{n}\right),\left[\xi_{1}: \cdots: \xi_{n}\right]\right)$ homogeneous coordinates, where $\left[\xi_{1}: \cdots:\right.$ $\left.\xi_{n}\right]$ are homogeneous coordinates of the dual projective space $P\left(\mathbb{R}^{n-1}\right)^{*}$. 
It is easy to show that $X \in K_{(x,[\xi])}$ if and only if $\sum_{i=1}^{n} \mu_{i} \xi_{i}=0$, where $d \tilde{\pi}(X)=\sum_{i=1}^{n} \mu_{i} \frac{\partial}{\partial x_{i}}$. An immersion $i: L \rightarrow P T^{*}(M)$ is said to be a Legendrian immersion if $\operatorname{dim} L=n$ and $d i_{q}\left(T_{q} L\right) \subset K_{i(q)}$ for any $q \in L$. We also call the map $\pi \circ i$ the Legendrian map and the set $W(i)=$ image $\pi \circ i$ the wave front of $i$. Moreover, $i$ (or, the image of $i$ ) is called the Legendrian lift of $W(i)$. The main tool of the theory of Legendrian singularities is the notion of generating families. Here we only consider local properties, we may assume that $M=\mathbb{R}^{n}$. Let $F:\left(\mathbb{R}^{k} \times \mathbb{R}^{n}, \mathbf{0}\right) \longrightarrow(\mathbb{R}, \mathbf{0})$ be a function germ. We say that $F$ is a Morse family of hypersurfaces if the mapping

$$
\Delta^{*} F=\left(F, \frac{\partial F}{\partial q_{1}}, \ldots, \frac{\partial F}{\partial q_{k}}\right):\left(\mathbb{R}^{k} \times \mathbb{R}^{n}, \mathbf{0}\right) \longrightarrow\left(\mathbb{R} \times \mathbb{R}^{k}, \mathbf{0}\right)
$$

is non-singular, where $(q, x)=\left(q_{1}, \ldots, q_{k}, x_{1}, \ldots, x_{n}\right) \in\left(\mathbb{R}^{k} \times \mathbb{R}^{n}, \mathbf{0}\right)$. In this case we have a smooth $(n-1)$-dimensional submanifold

$$
\Sigma_{*}(F)=\left\{(q, x) \in\left(\mathbb{R}^{k} \times \mathbb{R}^{n}, \mathbf{0}\right) \mid F(q, x)=\frac{\partial F}{\partial q_{1}}(q, x)=\cdots=\frac{\partial F}{\partial q_{k}}(q, x)=0\right\}
$$

and the map germ $\mathcal{L}_{F}:\left(\Sigma_{*}(F), \mathbf{0}\right) \longrightarrow P T^{*} \mathbb{R}^{n}$ defined by

$$
\mathcal{L}_{F}(q, x)=\left(x,\left[\frac{\partial F}{\partial x_{1}}(q, x): \cdots: \frac{\partial F}{\partial x_{n}}(q, x)\right]\right)
$$

is a Legendrian immersion germ. Then we have the following fundamental theorem of Arnol'dZakalyukin [1].

Proposition 8.1 All Legendrian submanifold germs in $P T^{*} \mathbb{R}^{n}$ are constructed by the above method.

We call $F$ a generating family of $\mathcal{L}_{F}\left(\Sigma_{*}(F)\right)$. Therefore the wave front is

$$
W\left(\mathcal{L}_{F}\right)=\left\{x \in \mathbb{R}^{n} \mid \exists q \in \mathbb{R}^{k} \text { such that } F(q, x)=\frac{\partial F}{\partial q_{1}}(q, x)=\cdots=\frac{\partial F}{\partial q_{k}}(q, x)=0\right\} .
$$

We now introduce an equivalence relation among Legendrian immersion germs. Let $i$ : $(L, p) \subset\left(P T^{*} \mathbb{R}^{n}, p\right)$ and $i^{\prime}:\left(L^{\prime}, p^{\prime}\right) \subset\left(P T^{*} \mathbb{R}^{n}, p^{\prime}\right)$ be Legendrian immersion germs. Then we say that $i$ and $i^{\prime}$ are Legendrian equivalent if there exists a contact diffeomorphism germ $H:\left(P T^{*} \mathbb{R}^{n}, p\right) \longrightarrow\left(P T^{*} \mathbb{R}^{n}, p^{\prime}\right)$ such that $H$ preserves fibers of $\pi$ and that $H(L)=L^{\prime}$. We can also define the notion of Legendrian stable map-germs analogous to Lagrangian stable map-germs. However, we do not use the definition, so that we omit it.

Since the Legendrian lift $i:(L, p) \subset\left(P T^{*} \mathbb{R}^{n}, p\right)$ is uniquely determined on the regular part of the wave front $W(i)$, we have the following significant property of Legendrian immersion germs:

Proposition 8.2 ([?]) Let $i:(L, p) \subset\left(P T^{*} \mathbb{R}^{n}, p\right)$ and $i^{\prime}:\left(L^{\prime}, p^{\prime}\right) \subset\left(P T^{*} \mathbb{R}^{n}, p^{\prime}\right)$ be Legendrian immersion germs such that regular sets of $\pi \circ i, \pi \circ i^{\prime}$ are dense respectively. Then $i, i^{\prime}$ are Legendrian equivalent if and only if wave front sets $W(i), W\left(i^{\prime}\right)$ are diffeomorphic as set germs.

The assumption in the above proposition is a generic condition for $i, i^{\prime}$. Specially, if $i, i^{\prime}$ are Legendrian stable, then these satisfy the assumption. We can interpret the Legendrian equivalence by using the notion of generating families. We denote $\mathcal{E}_{n}$ the local ring of function germs $\left(\mathbb{R}^{n}, \mathbf{0}\right) \longrightarrow \mathbb{R}$ with the unique maximal ideal $\mathfrak{M}_{n}=\left\{h \in \mathcal{E}_{n} \mid h(0)=0\right\}$. 
Let $F, G:\left(\mathbb{R}^{k} \times \mathbb{R}^{n}, \mathbf{0}\right) \longrightarrow(\mathbb{R}, \mathbf{0})$ be function germs. We say that $F$ and $G$ are $P$ - $\mathcal{K}$ equivalent if there exists a diffeomorphism germ $\Psi:\left(\mathbb{R}^{k} \times \mathbb{R}^{n}, \mathbf{0}\right) \longrightarrow\left(\mathbb{R}^{k} \times \mathbb{R}^{n}, \mathbf{0}\right)$ of the form $\Psi(x, u)=\left(\psi_{1}(q, x), \psi_{2}(x)\right)$ for $(q, x) \in\left(\mathbb{R}^{k} \times \mathbb{R}^{n}, \mathbf{0}\right)$ such that $\Psi^{*}\left(\langle F\rangle_{\mathcal{E}_{k+n}}\right)=\langle G\rangle_{\mathcal{E}_{k+n}}$. Here $\Psi^{*}: \mathcal{E}_{k+n} \longrightarrow \mathcal{E}_{k+n}$ is the pull back $\mathbb{R}$-algebra isomorphism defined by $\Psi^{*}(h)=h \circ \Psi$. If $n=0$, we simply say these germs are $\mathcal{K}$-equivalent.

Let $F:\left(\mathbb{R}^{k} \times \mathbb{R}^{3}, \mathbf{0}\right) \longrightarrow(\mathbb{R}, \mathbf{0})$ be a function germ. We say that $F$ is a $\mathcal{K}$-versal deformation of $f=F \mid \mathbb{R}^{k} \times\{\mathbf{0}\}$ if

$$
\mathcal{E}_{k}=T_{e}(\mathcal{K})(f)+\left\langle\frac{\partial F}{\partial x_{1}}\left|\mathbb{R}^{k} \times\{\mathbf{0}\}, \ldots, \frac{\partial F}{\partial x_{n}}\right| \mathbb{R}^{k} \times\{\mathbf{0}\}\right\rangle_{\mathbb{R}},
$$

where

$$
T_{e}(\mathcal{K})(f)=\left\langle\frac{\partial f}{\partial q_{1}}, \ldots, \frac{\partial f}{\partial q_{k}}, f\right\rangle_{\mathcal{E}_{k}}
$$

(See [15].)

The main result in Arnol'd-Zakalyukin's theory [1, 18] is the following:

Theorem 8.3 Let $F \in \mathfrak{M}_{k+n}, G \in \mathfrak{M}_{k^{\prime}+n}$ be Morse families of hypersurfaces. Then

(1) $\mathcal{L}_{F}\left(\Sigma_{*}(F)\right)$ and $\mathcal{L}_{G}\left(\Sigma_{*}(G)\right)$ are Legendrian equivalent if and only if $F$, G are stably $P-\mathcal{K}$ equivalent.

(2) $\mathcal{L}_{F}\left(\Sigma_{*}(F)\right)$ is Legendrian stable if and only if $F$ is a $\mathcal{K}$-versal deformation of $F \mid \mathbb{R}^{k} \times\{\mathbf{0}\}$.

The definition of the stably $P$ - $\mathcal{K}$-equivalence is similar to that of the stably $P$ - $\mathcal{R}^{+}$-equivalence. By the uniqueness result of the $\mathcal{K}$-versal deformation of a function germ, Proposition 8.2 and Theorem 8.3, we have the following classification result of Legendrian stable germs (cf., [6, Proposition A.4]). For any map germ $f:\left(\mathbb{R}^{n}, \mathbf{0}\right) \longrightarrow\left(\mathbb{R}^{p}, \mathbf{0}\right)$, we define the local ring of $f$ by $Q_{r}(f)=\mathcal{E}_{n} / f^{*}\left(\mathfrak{M}_{p}\right) \mathcal{E}_{n}+\mathfrak{M}_{n}^{r+1}$.

Proposition 8.4 Let $F, G:\left(\mathbb{R}^{k} \times \mathbb{R}^{n}, \mathbf{0}\right) \longrightarrow(\mathbb{R}, 0)$ be Morse families of hypersurfaces. Suppose that $\mathcal{L}(F), \mathcal{L}(G)$ are Legendrian stable. The the following conditions are equivalent:

(1) $\left(W\left(\mathcal{L}_{F}\right), \mathbf{0}\right)$ and $\left(W\left(\mathcal{L}_{G}\right), \mathbf{0}\right)$ are diffeomorphic as germs,

(2) $\mathcal{L}_{F}\left(\Sigma_{*}(F)\right)$ and $\mathcal{L}_{G}\left(\Sigma_{*}(G)\right)$ are Legendrian equivalent,

(3) $Q_{n+1}(f)$ and $Q_{n+1}(g)$ are isomorphic as $\mathbb{R}$-algebras,

where $f=F\left|\mathbb{R}^{k} \times\{\mathbf{0}\}, g=G\right| \mathbb{R}^{k} \times\{\mathbf{0}\}$.

We now apply the above theory of Legendrian singularities to our case. Firstly, we show the following.

Proposition 8.5 The extended lightcone height function $\widetilde{H}: U \times L C^{*} \longrightarrow \mathbb{R}$ is a Morse family of hypersurfaces.

Proof. Let $\boldsymbol{X}=\left(X_{0}, \ldots, X_{n}\right)$ and $\boldsymbol{v}=\left(v_{0}, \ldots, v_{n}\right) \in L C^{*}$, without loss of the generality, we assume that $v_{n}>0$, then $v_{0}=\sqrt{v_{1}^{2}+\cdots+v_{n}^{2}}$. Let $\Delta^{*} \widetilde{H}=\left(\widetilde{H}, \widetilde{H}_{u_{1}}, \ldots, \widetilde{H}_{u_{s}}\right)$, we now prove that a map $\Delta \widetilde{H}$ is submersive at $\left(u_{0}, \boldsymbol{v}_{0}\right) \in \Delta^{*} \widetilde{H}^{-1}(0)$. Its Jacobian matrix $J \Delta^{*} \widetilde{H}$ is

$$
J \Delta^{*} \widetilde{H}=\left(\begin{array}{c|c}
\left(\widetilde{H}_{u_{j}}\right)_{j=1, \ldots, s} & \left(\widetilde{H}_{v_{j}}\right)_{j=1, \ldots, n-1} \\
\hline\left(\widetilde{H}_{u_{i} u_{j}}\right)_{i, j=1, \ldots, s} & \left(\widetilde{H}_{u_{i} v_{j}}\right)_{i=1, \ldots, s, j=1, \ldots, n-1}
\end{array}\right)=:(* \mid B) .
$$


It is sufficient show that the rank of the matrix $B\left(u_{0}, \boldsymbol{v}_{0}\right)$ is $s+1$. By straightforward calculation, we have

$$
\begin{gathered}
\widetilde{H}_{v_{j}}(u, \boldsymbol{v})=-\frac{v_{j}}{v_{0}}+\frac{X_{j}}{v_{0}}-\sum_{k=1}^{n} \frac{v_{k} v_{j}}{v_{0}^{3}} X_{k}, \\
\widetilde{H}_{u_{i} v_{j}}(u, \boldsymbol{v})=-\frac{\left(X_{j}\right)_{u_{i}}}{v_{0}}-\sum_{k=1}^{n} \frac{v_{k} v_{j}}{v_{0}^{3}}\left(X_{k}\right)_{u_{i}},
\end{gathered}
$$

for $i=1, \ldots, s$ and $j=1, \ldots, n$. By the condition that $\widetilde{H}\left(u_{0}, \boldsymbol{v}_{0}\right)=\widetilde{H}_{u_{i}}\left(u_{0}, \boldsymbol{v}_{0}\right)=0$ for $i$, we have relations $\sum_{k=1}^{n} \frac{v_{0, k}}{v_{0,0}} X_{k}=X_{0}+v_{0,0}$ and $\sum_{k=1}^{n} \frac{v_{0, k}}{v_{0,0}}\left(X_{k}\right)_{u_{i}}=\left(X_{0}\right)_{u_{i}}$ where $\boldsymbol{v}_{0}=\left(v_{0,0}, \ldots, v_{0, n}\right)$. Therefore, the above formula is

$$
\begin{aligned}
\widetilde{H}_{v_{j}}\left(u_{0}, \boldsymbol{v}_{0}\right) & =\frac{1}{v_{0,0}}\left(X_{j}-2 v_{j}-X_{0} \frac{v_{0, j}}{v_{0,0}}\right), \\
\widetilde{H}_{u_{i} v_{j}}\left(u_{0}, \boldsymbol{v}_{0}\right) & =\frac{1}{v_{0,0}}\left(\left(X_{j}\right)_{u_{i}}-\left(X_{0}\right)_{u_{i}} \frac{v_{0, j}}{v_{0,0}}\right),
\end{aligned}
$$

for $i=1, \ldots, s$ and $j=1, \ldots, n$.

Since $\left\langle\boldsymbol{v}_{0}, \boldsymbol{v}_{0}\right\rangle=\left\langle\boldsymbol{v}_{0}, \boldsymbol{X}_{u_{i}}\right\rangle=0$ for $i=1, \ldots, s, \boldsymbol{v}_{0}$ and $\boldsymbol{X}_{u_{i}}\left(u_{0}\right)$ are belongs to $\operatorname{HP}\left(\boldsymbol{v}_{0}, 0\right)$. On the other hand, we have $\left\langle\boldsymbol{X}\left(u_{0}\right)-2 \boldsymbol{v}_{0}+2 v_{0,0} \mathbf{e}_{0}, \boldsymbol{v}_{0}\right\rangle=-2 v_{0,0}^{2} \neq 0$ where $\mathbf{e}_{0}=(1,0, \ldots, 0)$. So that vectors $\boldsymbol{X}\left(u_{0}\right)-2 \boldsymbol{v}_{0}+2 v_{0,0} \mathbf{e}_{0}, \boldsymbol{v}_{0}$ and $\boldsymbol{X}_{u_{i}}\left(u_{0}\right)$ (for $i=1, \ldots, s$ ) are linearly independent. Therefore the rank of following matrix

$$
\mathrm{C}=\left(\begin{array}{c}
\boldsymbol{v}_{0} \\
X-2 \boldsymbol{v}_{0}+2 v_{0,0} \mathbf{e}_{0} \\
X_{u_{1}} \\
\vdots \\
X_{u_{s}}
\end{array}\right)=\left(\begin{array}{cccc}
v_{0,0} & v_{0,1} & \cdots & v_{0, n} \\
X_{0} & X_{1}-2 v_{1} & \cdots & X_{n}-2 v_{n} \\
\left(X_{0}\right)_{u_{1}} & \left(X_{1}\right)_{u_{1}} & \cdots & \left(X_{n}\right)_{u_{1}} \\
\vdots & \vdots & \ddots & \vdots \\
\left(X_{0}\right)_{u_{s}} & \left(X_{1}\right)_{u_{s}} & \cdots & \left(X_{n}\right)_{u_{s}}
\end{array}\right)
$$

is $s+2$ at $\left(u_{0}, \boldsymbol{v}_{0}\right)$. We subtract the first law by multiplied by $X_{0} / v_{0,0}$ from the second raw, and we also subtract the first raw multiplied by $\left(X_{0}\right)_{u_{i}} / v_{0,0}$ from the $(2+i)$-th raw for $i=1, \ldots, s$. Then we have

$$
\mathrm{C}^{\prime}=\left(\begin{array}{c|c}
v_{0,0} & v_{0,1} \cdots v_{0, n} \\
\hline 0 & \\
\vdots & B\left(u_{0}, \boldsymbol{v}_{0}\right) \\
0 &
\end{array}\right)
$$

Therefore $\operatorname{rank} B\left(u_{0}, \boldsymbol{v}_{0}\right)=s+1$, this completes the proof.

It follows that we have the Legendrian submanifold germ $\left(\mathcal{L}_{\widetilde{H}}\left(\Sigma_{*}(\widetilde{H})\right), \bar{z}\right) \subset\left(P T^{*} L C^{*}, \bar{z}\right)$ such that $\pi\left(\mathcal{L}_{\widetilde{H}}\left(\Sigma_{*}(\widetilde{H})\right)\right)=\mathbb{L} \mathbb{P}\left(U \times S^{k-2}\right)$, which is called the Legendrian lift germ of the suspended lightcone pedal hypersurface germ $\mathbb{L} \mathbb{P}\left(U \times S^{k-2}\right)$.

Theorem 8.6 Let $\boldsymbol{X}_{i}:\left(U, \bar{u}_{i}\right) \longrightarrow\left(\mathbb{R}_{1}^{n+1}, p_{i}\right)$ be spacelike embedding germs of codimension $k$ $(i=1,2)$ and we write $M_{i}=\boldsymbol{X}_{i}(U)$. Suppose that both the Legendrian lift germs $\left(\mathcal{L}_{\widetilde{H}_{i}}\left(\Sigma_{*}\left(\widetilde{H}_{i}\right)\right), \bar{z}_{i}\right) \subset$ 
$\left(P T^{*} L C^{*}, \bar{z}_{i}\right)$ of the suspended lightcone pedal hypersurface germs $\left(\mathbb{L} \mathbb{P}_{i}\left(U_{i} \times S^{k-2}\right), \boldsymbol{v}_{i}\right)$ are Legendrian stable, where $\boldsymbol{v}_{i}=\mathbb{L} \mathbb{P}_{i}\left(\bar{u}_{i}, \bar{\mu}_{i}\right)$ and $\operatorname{THP}\left(M_{i},\left(\bar{u}_{i}, \bar{\mu}_{i}\right)\right)$ is the tangent lightlike hyperplane of $M_{i}$ at $p_{i}=\boldsymbol{X}\left(\bar{u}_{i}\right)$. Then the following conditions are equivalent:

(1) $K\left(M_{1}, \operatorname{THP}\left(M_{1},\left(\bar{u}_{1}, \bar{\mu}_{1}\right)\right) ; p_{1}\right)=K\left(M_{2}, \operatorname{THP}\left(M_{2},\left(\bar{u}_{2}, \bar{\mu}_{2}\right)\right) ; p_{2}\right)$.

(2) $\widetilde{h}_{1, \boldsymbol{v}_{1}}$ and $\widetilde{h}_{2, \boldsymbol{v}_{2}}$ are $\mathcal{K}$-equivalent.

(3) $\widetilde{H}_{1}$ and $\widetilde{H}_{2}$ are $P$-K-equivalent.

(4) $\left(\mathcal{L}_{\widetilde{H}_{1}}\left(\Sigma_{*}\left(\widetilde{H}_{1}\right)\right), \bar{z}_{1}\right)$ and $\left(\mathcal{L}_{\widetilde{H}_{2}}\left(\Sigma_{*}\left(\widetilde{H}_{2}\right)\right), \bar{z}_{2}\right)$ are Legendrian equivalent.

(5) $\left(\mathbb{L}_{1}\left(U_{1} \times S^{k-2}\right), \boldsymbol{v}_{1}\right)$ and $\left(\mathbb{L}_{2}\left(U_{2} \times S^{k-2}\right), \boldsymbol{v}_{2}\right)$ are diffeomorphic as set germs.

Proof. By Proposition 6.6, the conditions (1) and (2) are equivalent. By definition, the condition (3) implies the condition (2). By the uniqueness on the infinitesimally $\mathcal{K}$-versal deformation and Theorem 7.2, the condition (2) implies the condition (3). By the assertion (1) of Theorem 7.2, the conditions (3) and (4) are equivalent. The conditions (4) and (5) are equivalent as a consequence of Proposition 8.4. This completes the proof.

\section{$9 \quad$ Spacelike submanifolds with a parallel timelike normal vector field}

In this section we consider a special class of spacelike submanifolds with a parallel future directed timelike normal vector field, which contains several important examples. We say that $\boldsymbol{n}^{T}$ is parallel if $\boldsymbol{n}_{u_{i}}^{T}(i=1, \ldots, s)$ are tangent to $M=\boldsymbol{X}(U)$. Under this assumption, we can clarify the local geometric meaning of the lightcone curvature. For general $r>0, C M_{2}\left(\boldsymbol{n}^{T} ; r\right)$ is not necessarily spacelike even if it is a submanifold.

Proposition 9.1 Suppose that $\boldsymbol{n}^{T}$ is parallel along $M$. If $C M_{2}\left(\boldsymbol{n}^{T} ; r\right)$ is a submanifold,then it is spacelike.

Proof. Since $\left\langle\boldsymbol{n}^{T}, \boldsymbol{n}_{j}^{S}\right\rangle=0(j=1, \ldots, s)$, we have $\left\langle\boldsymbol{n}_{u_{i}}^{T}, \boldsymbol{n}_{j}^{S}\right\rangle=-\left\langle\boldsymbol{n}^{T}, \boldsymbol{n}_{j, u_{i}}^{S}\right\rangle$. By the assumption, $\boldsymbol{n}_{u_{i}}^{T}$ is tangent to $M=\boldsymbol{X}(U)$, so that we have $\left\langle\boldsymbol{n}^{T}, \boldsymbol{n}_{j, u_{i}}^{S}\right\rangle=0$. It follows that $\boldsymbol{n}_{j}^{S}(j=1, \ldots, s)$ are spacelike. By the proof of Proposition 5.1, we have

$$
\begin{aligned}
& \frac{\partial \overline{\boldsymbol{X}}\left(\boldsymbol{n}^{T}, \boldsymbol{N}^{S} ; r\right)}{\partial u_{i}}=\boldsymbol{X}_{u_{i}}(u)+r \sum_{j=1}^{k-1} \mu_{j} \boldsymbol{n}_{j, u_{i}}^{S}(u) \\
& \frac{\partial \overline{\boldsymbol{X}}\left(\boldsymbol{n}^{T}, \boldsymbol{N}^{S} ; r\right)}{\partial \mu_{j}}=r\left(\boldsymbol{n}_{j}^{S}(u)-\frac{\mu_{j}}{\mu_{1}} \boldsymbol{n}_{1}^{S}(u)\right)
\end{aligned}
$$

on the local coordinate neighborhood $U_{1}^{+}$. These are all spacelike vectors.

On the other local coordinate neighborhoods, we have the similar calculations to the above. This completes the proof.

We also have the following good properties for a parallel future directed timelike unit normal vector field $\boldsymbol{n}^{T}$. 
Proposition 9.2 Let $\boldsymbol{n}^{T}$ be a future directed timelike unit normal vector field along $M=\boldsymbol{X}(U)$ and $\left\{\boldsymbol{n}^{T}, \boldsymbol{n}_{1}^{S}, \ldots, \boldsymbol{n}_{k-1}^{S}\right\}$ a pseudo-orthonormal frame of the normal bundle $N(M)$. Then $\boldsymbol{N}$ is a unit normal vector field along $C M_{2}\left(\boldsymbol{n}^{T} ; r\right)$ if and only if $\boldsymbol{n}^{T}$ is parallel, where $\boldsymbol{N}^{S}(u, \mu)=$ $\sum_{j=1}^{k-1} \mu_{j} \boldsymbol{n}_{j}^{S}(u)$.

Proof. We consider the local coordinate $U_{1}^{+}$. Then we have

$$
\begin{aligned}
& \frac{\partial \overline{\boldsymbol{X}}\left(\boldsymbol{n}^{T}, \boldsymbol{N}^{S} ; r\right)}{\partial u_{i}}=\boldsymbol{X}_{u_{i}}(u)+r \sum_{j=1}^{k-1} \mu_{j} \boldsymbol{n}_{j, u_{i}}^{S}(u) \\
& \frac{\partial \overline{\boldsymbol{X}}\left(\boldsymbol{n}^{T}, \boldsymbol{N}^{S} ; r\right)}{\partial \mu_{j}}=r\left(\boldsymbol{n}_{j}^{S}(u)-\frac{\mu_{j}}{\mu_{1}} \boldsymbol{n}_{1}^{S}(u)\right) .
\end{aligned}
$$

By a straightforward calculation, we can show that $\boldsymbol{N}^{S}$ is always pseudo-orthogonal to $\partial \overline{\boldsymbol{X}}\left(\boldsymbol{n}^{T}, \boldsymbol{N}^{S} ; r\right) / \partial \mu_{j}$. Moreover, we have the following calculations:

$$
\begin{aligned}
& \left\langle\boldsymbol{N}^{S}(u, \mu), \boldsymbol{X}_{u_{i}}(u)+r \sum_{j=1}^{k-1} \mu_{j} \boldsymbol{n}_{j, u_{i}}^{S}(u)\right\rangle \\
& =r\left(\sum_{j=1}^{k-1} \mu_{j}\left\langle\boldsymbol{n}^{T}(u), \boldsymbol{n}_{j, u_{i}}^{S}(u)\right\rangle+\left\langle\sum_{j=1}^{k-1} \mu_{j} \boldsymbol{n}_{j}^{S}(u), \sum_{j=1}^{k-1} \mu_{j} \boldsymbol{n}_{j, u_{i}}^{S}(u)\right\rangle\right) \\
& =r\left(\sum_{j=1}^{k-1} \mu_{j}\left\langle\boldsymbol{n}^{T}(u), \boldsymbol{n}_{j, u_{i}}^{S}(u)\right\rangle\right)
\end{aligned}
$$

On the other hand, $r\left(\sum_{j=1}^{k-1} \mu_{j}\left\langle\boldsymbol{n}^{T}(u), \boldsymbol{n}_{j, u_{i}}^{S}(u)\right\rangle\right)=0$ for any $\mu=\left(\mu_{1}, \ldots, \mu_{k-1}\right) \in S^{k-2}$ if and only if $\left\langle\boldsymbol{n}^{T}(u), \boldsymbol{n}_{j, u_{i}}^{S}(u)\right\rangle=0$ for $j=1, \ldots, k-1$. The last condition is equivalent to the condition $\left\langle\boldsymbol{n}_{u_{i}}^{T}(u), \boldsymbol{n}_{j}^{S}(u)\right\rangle=0$ for $j=1, \ldots, k-1$. Since $\left\{\boldsymbol{n}^{T}, \boldsymbol{n}_{1}^{S}, \ldots, \boldsymbol{n}_{k-1}^{S}\right\}$ a pseudoorthonormal frame of the normal bundle $N(M)$, the last condition is equivalent to the condition that $\boldsymbol{n}^{T}$ is parallel. This completes the proof.

We have the following corollary.

Corollary 9.3 Under the same notations as the above proposition, $\boldsymbol{n}^{T} \pm \boldsymbol{N}^{S}$ are lightlike normal vector fields along $C M_{2}\left(\boldsymbol{n}^{T} ; r\right)$ if and only if $\boldsymbol{n}^{T}$ is parallel.

Thus, we have the suspended normalized Gauss map $\widetilde{\mathbb{L} G}: U \times S^{k-2} \longrightarrow S_{+}^{n-1}$ and the suspended lightcone pedal hypersurface $\mathbb{L} \mathbb{P}: U \times S^{k-2} \longrightarrow L C^{*}$ are respectively the lightcone Gauss map and the lightcone pedal of $C M_{2}\left(\boldsymbol{n}^{T} ; r\right)$ as a spacelike submanifold of codimension two defined in [11]. In this case we consider the family of lightcone height functions (cf., [11])

$$
H^{C M}: U \times S^{k-2} \times S_{+}^{n-1} \longrightarrow \mathbb{R}
$$

defined by $H^{C M}(u, \mu, \boldsymbol{w})=\left\langle\overline{\boldsymbol{X}}\left(\boldsymbol{n}^{T}, \boldsymbol{N}^{S} ; r\right)(u, \mu), \boldsymbol{w}\right\rangle$. We also have the family of extended lightcone height functions

$$
\widetilde{H}^{C M}: U \times S^{k-2} \times L C^{*} \longrightarrow \mathbb{R}
$$

defined by $\widetilde{H}^{C M}(u, \mu, \boldsymbol{v})=\left\langle\overline{\boldsymbol{X}}\left(\boldsymbol{n}^{T}, \boldsymbol{N}^{S} ; r\right)(u, \mu), \widetilde{\boldsymbol{v}}\right\rangle-v_{0}$. Since $C M_{2}\left(\boldsymbol{n}^{T} ; r\right)$ is codimension two, we can apply the results in [11]. Especially, by Propositions 4.2 and 5.1 in [11], we have the following proposition. 
Proposition 9.4 Suppose that $\boldsymbol{n}^{T}$ is parallel. Then we have the following:

(1) $\left(\partial H^{C M} / \partial u_{i}\right)\left(\bar{u}_{0}, \bar{\mu}_{0}, \boldsymbol{w}_{0}\right)=\left(\partial H^{C M} / \partial \mu_{j}\right)\left(\bar{u}_{0}, \bar{\mu}_{0}, \boldsymbol{w}_{0}\right)=0(i=1, \ldots, s, j=1, \ldots k-1)$ if and only if $\boldsymbol{w}_{0}=\widetilde{\mathbb{L}}^{ \pm}\left(\bar{u}_{0}, \bar{\mu}_{0}\right)$.

(2) $\widetilde{H}^{C M}\left(\bar{u}_{0}, \bar{\mu}_{0}, \boldsymbol{v}_{0}\right)=\left(\partial \widetilde{H}^{C M} / \partial u_{i}\right)\left(\bar{u}_{0}, \bar{\mu}_{0}, \boldsymbol{v}_{0}\right)=\left(\partial \widetilde{H}^{C M} / \partial \mu_{j}\right)\left(\bar{u}_{0}, \bar{\mu}_{0}, \boldsymbol{v}_{0}\right)=0(i=1, \ldots, s, j=$ $1, \ldots k-1)$ if and only if $\boldsymbol{v}_{0}=\mathbb{L} \mathbb{P}^{ \pm}\left(\bar{u}_{0}, \bar{\mu}_{0}\right)$.

(3) We denote that $h_{\boldsymbol{w}}^{C M}\left(\bar{u}_{0}, \bar{\mu}_{0}\right)=H^{C M}\left(\bar{u}_{0}, \bar{\mu}_{0}, \boldsymbol{w}\right)$ and $\widetilde{h}_{\boldsymbol{v}}^{C M}\left(\bar{u}_{0}, \bar{\mu}_{0}\right)=\widetilde{H}^{C M}\left(\bar{u}_{0}, \bar{\mu}_{0}, \boldsymbol{v}\right)$. Then the following conditions are equivalent:

(a) $\left(p_{0}, \boldsymbol{\xi}_{0}\right)=\overline{\boldsymbol{X}}\left(\boldsymbol{n}^{T}, \boldsymbol{N}^{s} ; r\right)\left(\bar{u}_{0}, \bar{\mu}_{0}\right) \in C M\left(\boldsymbol{n}^{T} ; r\right)$ is a lightlike parabolic point of $C M\left(\boldsymbol{n}^{T} ; r\right)$.

(b) $\operatorname{det} \operatorname{Hess}\left(h_{\widetilde{\boldsymbol{v}}_{0}}^{C M}\right)\left(\bar{u}_{0}, \bar{\mu}_{0}\right)=0$,

(c) det $\operatorname{Hess}\left(\widetilde{h}_{\boldsymbol{v}_{0}}^{C M}\right)\left(\bar{u}_{0}, \bar{\mu}_{0}\right)=0$,

where $\widetilde{\boldsymbol{v}}_{0}=\widetilde{\mathbb{L}}^{ \pm}\left(\bar{u}_{0}, \bar{\mu}_{0}\right)$ and $\boldsymbol{v}_{0}=\mathbb{L} \mathbb{P}^{ \pm}\left(\bar{u}_{0}, \bar{\mu}_{0}\right)$.

The assertions (1) and (2) of the above proposition describe that

$$
\begin{aligned}
C\left(H^{C M}\right) & =\left\{\left((u, \mu), \widetilde{\mathbb{L} \mathbb{G}^{ \pm}}(u, \mu)\right) \mid(u, \mu) \in U \times S^{k-2}\right\}, \\
W\left(\mathcal{L}_{\widetilde{H}^{C M}}\right) & =\left\{\mathbb{L} \mathbb{P}^{ \pm}(u, \mu) \mid(u, \mu) \in U \times S^{k-2}\right\} .
\end{aligned}
$$

Moreover, the assertion (3) means that the both of the singularities of $\widetilde{\mathbb{L} G}{ }^{ \pm}, \mathbb{L P}^{ \pm}$are the set of lightlike parabolic points of $C M\left(\boldsymbol{n}^{T} ; r\right)$. As a consequence, we have the following theorem.

Theorem 9.5 Let $\boldsymbol{X}_{i}:\left(U, \bar{u}_{i}\right) \longrightarrow\left(\mathbb{R}_{1}^{n+1}, p_{i}\right)$ be spacelike embedding germs of codimension $k$ $(i=1,2)$ and we write $M_{i}=\boldsymbol{X}_{i}(U)$. Suppose that both the Legendrian lift germs

$$
\left(\mathcal{L}_{\widetilde{H}_{i}}\left(\Sigma_{*}\left(\widetilde{H}_{i}\right)\right), \bar{z}_{i}\right) \subset\left(P T^{*} L C^{*}, \bar{z}_{i}\right)
$$

of the suspended lightcone pedal hypersurface germs $\left(\mathbb{L}_{i}\left(U_{i} \times S^{k-2}\right), \boldsymbol{v}_{i}\right)$ are Legendrian stable, where $\boldsymbol{v}_{i}=\mathbb{L} \mathbb{P}_{i}\left(\bar{u}_{i}, \bar{\mu}_{i}\right)$ and THP $\left(M_{i},\left(\bar{u}_{i}, \bar{\mu}_{i}\right)\right)$ is the tangent lightlike hyperplane of $M_{i}$ at $p_{i}=$ $\boldsymbol{X}\left(\bar{u}_{i}\right)$. Moreover, we assume that $\boldsymbol{n}_{i}^{T}$ are parallel and THP $\left.\left(C M_{2}\left(\boldsymbol{n}_{i}^{T} ; r\right),\left(\bar{u}_{i}, \bar{\mu}_{i}\right)\right) ; p_{i}+r \boldsymbol{\xi}_{i}\right)$ is the tangent lightlike hyperplane of the codimension two spacelike canal submanifold $C M_{2}\left(\boldsymbol{n}_{i}^{T} ; r\right)$ at $p_{i}+r \boldsymbol{\xi}_{i}=\overline{\boldsymbol{X}}\left(\boldsymbol{n}^{T}, \boldsymbol{N}^{S} ; r\right)\left(\bar{u}_{i}, \bar{\mu}_{i}\right)$. Then the following conditions are equivalent:

(1) $K\left(M_{1}, \operatorname{THP}\left(M_{1},\left(\bar{u}_{1}, \bar{\mu}_{2}\right)\right) ; p_{1}\right)=K\left(M_{2}, T H P\left(M_{2},\left(\bar{u}_{2}, \bar{\mu}_{2}\right)\right) ; p_{2}\right)$.

(2) $\widetilde{h}_{1, \boldsymbol{v}_{1}}$ and $\widetilde{h}_{2, \boldsymbol{v}_{2}}$ are $\mathcal{K}$-equivalent.

(3) $\widetilde{H}_{1}$ and $\widetilde{H}_{2}$ are P-K-equivalent.

(4) $\left(\mathcal{L}_{\widetilde{H}_{1}}\left(\Sigma_{*}\left(\widetilde{H}_{1}\right)\right), \bar{z}_{1}\right)$ and $\left(\mathcal{L}_{\widetilde{H}_{2}}\left(\Sigma_{*}\left(\widetilde{H}_{2}\right)\right), \bar{z}_{2}\right)$ are Legendrian equivalent.

(5) $\left(\mathbb{L} \mathbb{P}_{1}\left(U_{1} \times S^{k-2}\right), \boldsymbol{v}_{1}\right)$ and $\left(\mathbb{L}_{\mathbb{P}_{2}}\left(U_{2} \times S^{k-2}\right), \boldsymbol{v}_{2}\right)$ are diffeomorphic as set germs.

(6) $K\left(C M_{2}\left(\boldsymbol{n}_{1}^{T} ; r\right), \operatorname{THP}\left(C M_{2}\left(\boldsymbol{n}_{1}^{T} ; r\right),\left(\bar{u}_{1}, \bar{\mu}_{1}\right)\right) ; p_{1}+r \boldsymbol{\xi}_{1}\right)$

$$
=K\left(C M_{2}\left(\boldsymbol{n}_{2}^{T} ; r\right), T H P\left(C M_{2}\left(\boldsymbol{n}_{2}^{T} ; r\right),\left(\bar{u}_{2}, \bar{\mu}_{2}\right)\right) ; p_{2}+r \boldsymbol{\xi}_{2}\right) .
$$

(7) $\widetilde{h}_{\boldsymbol{v}_{1}}^{C M_{1}}$ and $\widetilde{h}_{\boldsymbol{v}_{2}}^{C M_{2}}$ are $\mathcal{K}$-equivalent.

(8) $\widetilde{H}^{C M_{1}}$ and $\widetilde{H}^{C M_{2}}$ are P-K-equivalent.

(9) $\left(\mathcal{L}_{\widetilde{H}^{C M_{1}}}\left(\Sigma_{*}\left(\widetilde{H}^{C M_{1}}\right)\right), z_{1}^{*}\right)$ and $\left(\mathcal{L}_{\widetilde{H}^{C M_{2}}}\left(\Sigma_{*}\left(\widetilde{H}^{C M_{2}}\right)\right), z_{2}^{*}\right)$ are Legendrian equivalent. 
Proof. It has been already shown in Theorem 8.6 that the conditions $(1),(2),(3),(4),(5)$ are equivalent. As a special case of Theorem 8.6, the conditions $(6),(7),(8),(9)$ are also equivalent. Since the suspended lightcone pedal hypersurfaces $\left(\mathbb{L P}_{i}\left(U_{i} \times S^{k-2}\right), \boldsymbol{v}_{i}\right)$ are wavefront sets of $\mathcal{L}_{\widetilde{H}^{C M}}$, the condition (5) is equivalent to the condition (9). This completes the proof.

\section{Spacelike curves in Lorentz-Minkowski 4-space}

In this section we consider spacelike curves in $\mathbb{R}_{1}^{4}$ as the simplest case of the previous results. Let $\gamma: I \longrightarrow \mathbb{R}_{1}^{4}$ be a spacelike curve with $\left\|\gamma^{\prime \prime}(s)\right\| \neq 0$. In this case we write $C=\gamma(I)$ instead of $M=\gamma(I)$. Since $\left\|\gamma^{\prime}(s)\right\|>0$, we can reparameterize it by the arc-length s. So we have the unit tangent vector $\boldsymbol{t}(s)=\gamma^{\prime}(s)$ of $\gamma(s)$. Moreover we have two unit normal vectors $\boldsymbol{n}_{1}(s)=\frac{\boldsymbol{\gamma}^{\prime \prime}(s)}{\kappa_{1}(s)}, \boldsymbol{n}_{2}(s)=\frac{\boldsymbol{n}_{1}^{\prime}(s)+\delta_{1} \kappa_{1}(s) \boldsymbol{t}(s)}{\left\|\boldsymbol{n}_{1}^{\prime}(s)+\delta_{1} \kappa_{1}(s) \boldsymbol{t}(s)\right\|}$ under the conditions that $\kappa_{1}(s)=\left\|\boldsymbol{\gamma}^{\prime \prime}(s)\right\| \neq 0$, $\kappa_{2}(s)=\left\|\boldsymbol{n}_{1}^{\prime}(s)+\delta k_{1}(s) \boldsymbol{t}(s)\right\| \neq 0$, where $\delta_{i}=\operatorname{sign}\left(\boldsymbol{n}_{i}(s)\right)$ and $\operatorname{sign}\left(\boldsymbol{n}_{i}(s)\right)$ is the signature of $\boldsymbol{n}_{i}(s)(i=1,2,3)$. Then we have another unit normal vector field $\boldsymbol{n}_{3}(s)$ defined by $\boldsymbol{n}_{3}(s)=\boldsymbol{t}(s) \wedge$ $\boldsymbol{n}_{1}(s) \wedge \boldsymbol{n}_{2}(s)$. Therefore we can construct a pseudo-orthogonal frame $\left\{\boldsymbol{t}(s), \boldsymbol{n}_{1}(s), \boldsymbol{n}_{2}(s), \boldsymbol{n}_{3}(s)\right\}$, which satisfies the Frenet-Serret type formulae:

$$
\left\{\begin{array}{l}
\boldsymbol{t}^{\prime}(s)=\kappa_{1}(s) \boldsymbol{n}_{1}(s) \\
\boldsymbol{n}_{1}^{\prime}(s)=-\delta_{1} \kappa_{1}(s) \boldsymbol{t}(s)+\kappa_{2}(s) \boldsymbol{n}_{2}(s) \\
\boldsymbol{n}_{2}^{\prime}(s)=\delta_{3} \kappa_{2}(s) \boldsymbol{n}_{1}(s)+\kappa_{3}(s) \boldsymbol{n}_{3}(s) \\
\boldsymbol{n}_{3}^{\prime}(s)=\delta_{1} \kappa_{3}(s) \boldsymbol{n}_{2}(s)
\end{array}\right.
$$

where $\kappa_{2}(s)=\delta_{2}\left\langle\boldsymbol{n}_{1}^{\prime}(s), \boldsymbol{n}_{2}(s)\right\rangle$ and $\kappa_{3}(s)=\delta_{3}\left\langle\boldsymbol{n}_{2}^{\prime}(s), \boldsymbol{n}_{3}(s)\right\rangle$. Since $\boldsymbol{t}(s)$ is spacelike, we distinguish the following three cases:

Case 1: $\boldsymbol{n}_{1}(s)$ is timelike, that is, $\delta_{1}=-1$ and $\delta_{2}=\delta_{3}=1$.

Case 2: $\boldsymbol{n}_{2}(s)$ is timelike, that is, $\delta_{2}=-1$ and $\delta_{1}=\delta_{3}=1$.

Case 3: $\boldsymbol{n}_{3}(s)$ is timelike, that is, $\delta_{3}=-1$ and $\delta_{1}=\delta_{2}=1$.

We consider the lightlike hypersurface along $C$, and calculate the Lorentzian distance-squared function on $C$ which is useful for the study the singularities of lightlike hypersurfaces in the each case.

\subsection{Case 1}

Suppose that $\boldsymbol{n}_{1}(s)$ is timelike. In this case we adopt $\boldsymbol{n}^{T}(s)=\boldsymbol{n}_{1}(s)$. Then we have the pseudo-orthogonal frame

$$
\left\{\boldsymbol{t}(s), \boldsymbol{n}^{T}(s), \boldsymbol{n}_{2}(s), \boldsymbol{n}_{3}(s)\right\},
$$

$\delta_{1}=-1$ and $\delta_{2}=\delta_{3}=1$, which satisfies the following Frenet-Serret type formulae:

$$
\left\{\begin{array}{l}
\boldsymbol{t}^{\prime}(s)=\kappa_{1}(s) \boldsymbol{n}^{T}(s) \\
\boldsymbol{n}^{T^{\prime}}(s)=\kappa_{1}(s) \boldsymbol{t}(s)+\kappa_{2}(s) \boldsymbol{n}_{2}(s) \\
\boldsymbol{n}_{2}^{\prime}(s)=\kappa_{2}(s) \boldsymbol{n}^{T}(s)+\kappa_{3}(s) \boldsymbol{n}_{3}(s) \\
\boldsymbol{n}_{3}^{\prime}(s)=-\kappa_{3}(s) \boldsymbol{n}_{2}(s)
\end{array}\right.
$$

Since $N_{1}(C)\left[\boldsymbol{n}^{T}\right]$ is parametrized by

$$
N_{1}(C)\left[\boldsymbol{n}^{T}\right]=\left\{(\boldsymbol{\gamma}(s), \boldsymbol{\xi}) \in \boldsymbol{\gamma}^{*} T \mathbb{R}_{1}^{4} \mid \boldsymbol{\xi}=\cos \theta \boldsymbol{n}_{2}(s)+\sin \theta \boldsymbol{n}_{3}(s) \in N_{\gamma(s)}(C), s \in I, \theta \in[-\pi, \pi)\right\},
$$


the lightcone Gauss map is given by

$$
\widetilde{\mathbb{L} G}(s, \theta)=\widetilde{\mathbb{L} G}\left(\boldsymbol{n}^{T}, \boldsymbol{N}^{S}\right)(s, \theta),
$$

where $\boldsymbol{N}^{S}(s, \theta)=\boldsymbol{n}^{T}(s)+\cos \theta \boldsymbol{n}_{2}(s)+\sin \theta \boldsymbol{n}_{3}(s)$. Moreover, the lightcone pedal is $\mathbb{L} \mathbb{P}(s, \theta)=$ $\langle\gamma(s), \widetilde{\mathbb{L} G}(s, \theta)\rangle \widetilde{\mathbb{L} \mathbb{G}}(s, \theta)$.

We now consider the lightcone height function $H: I \times S_{+}^{2} \longrightarrow \mathbb{R}$ defined by $H(s, \boldsymbol{v})=$ $\langle\gamma(s), \boldsymbol{v}\rangle$. In the general case, we have shown that

$$
C(H)=\{(s, \widetilde{\mathbb{L} G}(s, \theta)) \mid(s, \theta) \in I \times[-\pi, \pi)\} .
$$

By the Frenet-Serret type formulae, we have $h_{\boldsymbol{v}}^{\prime \prime}(s)=\left\langle\kappa_{1}(s) \boldsymbol{n}_{1}(s), \boldsymbol{v}\right\rangle$. For $\boldsymbol{v}=\widetilde{\mathbb{L} \mathbb{G}}(s, \theta)$, we have

$$
h_{\boldsymbol{v}}^{\prime \prime}(s)=\left\langle\kappa_{1}(s) \boldsymbol{n}_{1}(s), \widetilde{\mathbb{L} \mathbb{G}}(s, \theta)\right\rangle=-\ell_{0}(s) \kappa_{1}(s, \theta) \neq 0,
$$

where $\ell_{0}(s, \theta)$ is the first component of $\boldsymbol{n}^{T}(s)+\cos \theta \boldsymbol{n}_{2}(s)+\sin \theta \boldsymbol{n}_{3}(s)$. Therefore, the lightcone Gauss map and the lightcone pedal are non-singular.

\subsection{Case 2}

Suppose that $\boldsymbol{n}_{2}(s)$ is timelike. Then we adopt $\boldsymbol{n}^{T}(s)=\boldsymbol{n}_{2}(s)$. We have a pseudo-orthogonal frame $\left\{\boldsymbol{t}(s), \boldsymbol{n}^{T}(s), \boldsymbol{n}_{1}(s), \boldsymbol{n}_{3}(s)\right\}, \delta_{2}=-1$ and $\delta_{1}=\delta_{3}=1$, which satisfies the following FrenetSerret type formulae:

$$
\left\{\begin{array}{l}
\boldsymbol{t}^{\prime}(s)=\kappa_{1}(s) \boldsymbol{n}_{1}(s) \\
\boldsymbol{b}_{1}^{\prime}(s)=-\kappa_{1}(s) \boldsymbol{t}(s)+\kappa_{2}(s) \boldsymbol{n}^{T}(s) \\
\boldsymbol{n}^{T^{\prime}}(s)=\kappa_{2}(s) \boldsymbol{n}_{1}(s)+\kappa_{3}(s) \boldsymbol{n}_{3}(s) \\
\boldsymbol{b}_{2}^{\prime}(s)=\kappa_{3}(s) \boldsymbol{n}^{T}(s)
\end{array}\right.
$$

Here, $N_{1}(C)\left[\boldsymbol{n}^{T}\right]$ is parametrized by

$N_{1}(C)\left[\boldsymbol{n}^{T}\right]=\left\{(\boldsymbol{\gamma}(s), \boldsymbol{\xi}) \in \boldsymbol{\gamma}^{*} T \mathbb{R}_{1}^{4} \mid \boldsymbol{\xi}=\cos \theta \boldsymbol{n}_{1}(s)+\sin \theta \boldsymbol{n}_{3}(s) \in N_{\boldsymbol{\gamma}(s)}(C), s \in I, \theta \in[-\pi, \pi)\right\}$,

so that the lightcone Gauss map and the lightcone pedal are given by

$$
\widetilde{\mathbb{L} G}(s, \theta)=\widetilde{\mathbb{L} G}\left(\boldsymbol{n}^{T}, \boldsymbol{N}^{S}\right)(s, \theta),
$$

where $\boldsymbol{N}^{S}(s, \theta)=\boldsymbol{n}^{T}(s)+\cos \theta \boldsymbol{n}_{1}(s)+\sin \theta \boldsymbol{n}_{3}(s)$ and $\mathbb{L} \mathbb{P}(s, \theta)=\langle\boldsymbol{\gamma}(s), \widetilde{\mathbb{L} \mathbb{G}}(s, \theta)\rangle \widetilde{\mathbb{L} \mathbb{G}}(s, \theta)$. We also have $C(H)=\{(s, \widetilde{\mathbb{L} G}(s, \theta)) \mid(s, \theta) \in I \times[0,2 \pi)\}$ for the lightcone height function $H: I \times S_{+}^{2} \longrightarrow \mathbb{R}$. Moreover we have the following proposition.

Proposition 10.1 For $\boldsymbol{v}_{0} \in S_{+}^{2}$, we have the following:

(1) $h_{\boldsymbol{v}_{0}}^{\prime}\left(s_{0}\right)=0$ if and only if there exists $\theta_{0} \in[-\pi . \pi)$ such that $\boldsymbol{v}_{0}=\widetilde{\mathbb{L} \mathbb{G}}\left(s_{0}, \theta_{0}\right)$.

(2) $h_{\boldsymbol{v}_{0}}^{\prime}\left(s_{0}\right)=h_{\boldsymbol{v}_{0}}^{\prime \prime}(s)=0$ if and only if there $\boldsymbol{v}_{0}=\widetilde{\mathbb{L} \mathbb{G}}\left(s_{0}, \pm \pi / 2\right)$.

(3) $h_{\boldsymbol{v}_{0}}^{\prime}\left(s_{0}\right)=h_{\boldsymbol{v}_{0}}^{\prime \prime}\left(s_{0}\right)=h_{\boldsymbol{v}_{0}}^{\prime \prime \prime}\left(s_{0}\right)=0$ if and only if $\boldsymbol{v}_{0}=\widetilde{\mathbb{L} G}\left(s_{0}, \pm \pi / 2\right)$ and $\kappa_{2}\left(s_{0}\right)=0$.

(4) $h_{\boldsymbol{v}_{0}}^{\prime}\left(s_{0}\right)=h_{\boldsymbol{v}_{0}}^{\prime \prime}\left(s_{0}\right)=h_{\boldsymbol{v}_{0}}^{\prime \prime \prime}\left(s_{0}\right)=h_{\boldsymbol{v}_{0}}^{(4)}\left(s_{0}\right)=0$ if and only if $\boldsymbol{v}_{0}=\widetilde{\mathbb{L} \mathbb{G}}\left(s_{0}, \pm \pi / 2\right), \kappa_{2}\left(s_{0}\right)=0$ and $\kappa_{2}^{\prime}\left(s_{0}\right)=0$. 
Proof. By definition and the Frenet-Serret type formulae, we have

(a) $h_{\boldsymbol{v}}^{\prime}=\langle\boldsymbol{t}, \boldsymbol{v}\rangle$

(b) $h_{\boldsymbol{v}}^{\prime \prime}=\left\langle\kappa_{1} \boldsymbol{n}_{1}, \boldsymbol{v}\right\rangle$

(c) $h_{\boldsymbol{v}}^{\prime \prime \prime}=\left\langle\kappa_{1}^{\prime} \boldsymbol{n}_{1}-\kappa_{1}^{2} \boldsymbol{t}+\kappa_{1} \kappa_{2} \boldsymbol{n}^{T}, \boldsymbol{v}\right\rangle$

(d) $h_{\boldsymbol{v}}^{(4)}=\left\langle\left(\kappa_{1}^{\prime \prime}-\kappa_{1}^{3}+\kappa_{1} \kappa_{2}^{2}\right) \boldsymbol{n}_{1}-3 \kappa_{1}^{\prime} \kappa_{1} \boldsymbol{t}+\left(2 \kappa_{1}^{\prime} \kappa_{2}+\kappa_{1} \kappa_{2}^{\prime}\right) \boldsymbol{n}^{T}+\kappa_{1} \kappa_{2} \kappa_{3} \boldsymbol{n}_{3}, \boldsymbol{v}\right\rangle$.

By Proposition 4.1, we have the assertion (1). Suppose that $\boldsymbol{v}_{0}=\widetilde{\mathbb{L} \mathbb{G}}\left(s_{0}, \theta_{0}\right)$. By the above formula (b), we have $h_{\boldsymbol{v}_{0}}^{\prime \prime}\left(s_{0}\right)=0$ if and only if

$$
0=\left\langle\kappa_{1}\left(s_{0}\right) \boldsymbol{n}_{1}\left(s_{0}\right), \boldsymbol{v}_{0}\right\rangle=\kappa_{1}\left(s_{0}\right) \ell_{0}\left(s, \theta_{0}\right) \cos \theta_{0},
$$

where $\ell_{0}(s, \theta)$ is the first component of $\boldsymbol{n}^{T}(s)+\cos \theta \boldsymbol{n}_{1}(s)+\sin \theta \boldsymbol{n}_{3}(s)$. Since $\kappa_{1}(s) \ell_{0}(s, \theta) \neq 0$, we have $\cos \theta_{0}=0$. Therefore, $h_{\boldsymbol{v}_{0}}^{\prime}\left(s_{0}\right)=h_{\boldsymbol{v}_{0}}^{\prime \prime}(s)=0$ if and only if $\boldsymbol{v}_{0}=\widetilde{\mathbb{L} \mathbb{G}}\left(s_{0}, \pm \pi / 2\right)=$ $\ell_{0}\left(s_{0}, \pm \pi / 2\right)\left(\boldsymbol{n}^{T}\left(s_{0}\right) \pm \boldsymbol{n}_{3}\left(s_{0}\right)\right)$. By the above formula (c), the assertion (3) holds. By the similar arguments to the above cases we can show that the assertion (4) holds.

\subsection{Case 3}

Suppose that $\boldsymbol{n}_{3}(s)$ is timelike. Then we adopt $\boldsymbol{n}^{T}(s)=\boldsymbol{n}_{3}(s)$ and we have a pseudo-orthogonal frame $\left\{\boldsymbol{t}(s), \boldsymbol{n}^{T}(s), \boldsymbol{n}_{1}(s), \boldsymbol{n}_{2}(s)\right\}$ and $\delta_{3}=-1$ and $\delta_{1}=\delta_{2}=1$, which satisfies the following Frenet-Serret type formulae:

$$
\left\{\begin{array}{l}
\boldsymbol{t}^{\prime}(s)=\kappa_{1}(s) \boldsymbol{n}_{1}(s) \\
\boldsymbol{b}_{1}^{\prime}(s)=-\kappa_{1}(s) \boldsymbol{t}(s)+\kappa_{2}(s) \boldsymbol{n}_{2}(s) \\
\boldsymbol{b}_{2}^{\prime}(s)=-\kappa_{2}(s) \boldsymbol{n}_{1}(s)+\kappa_{3}(s) \boldsymbol{n}^{T}(s) \\
\boldsymbol{n}^{T^{\prime}}(s)=\kappa_{3}(s) \boldsymbol{n}_{2}(s)
\end{array}\right.
$$

Here, $N_{1}(C)\left[\boldsymbol{n}^{T}\right]$ is parametrized by

$N_{1}(C)\left[\boldsymbol{n}^{T}\right]=\left\{(\boldsymbol{\gamma}(s), \boldsymbol{\xi}) \in \boldsymbol{\gamma}^{*} T \mathbb{R}_{1}^{4} \mid \boldsymbol{\xi}=\cos \theta \boldsymbol{n}_{1}(s)+\sin \theta \boldsymbol{n}_{2}(s) \in N_{\gamma(s)}(C), s \in I, \theta \in[-\pi, \pi)\right\}$,

so that the lightcone Gauss map and the lightcone pedal are given by

$$
\widetilde{\mathbb{L} G}(s, \theta)=\widetilde{\mathbb{L} G}\left(\boldsymbol{n}^{T}, \boldsymbol{N}^{S}\right)(s, \theta),
$$

where $\boldsymbol{N}^{S}(s, \theta)=\boldsymbol{n}^{T}(s)+\cos \theta \boldsymbol{n}_{1}(s)+\sin \theta \boldsymbol{n}_{2}(s)$ and $\mathbb{L} \mathbb{P}(s, \theta)=\langle\gamma(s), \widetilde{\mathbb{L} \mathbb{G}}(s, \theta)\rangle \widetilde{\mathbb{L} G}(s, \theta)$. We also have $C(H)=\{(s, \widetilde{\mathbb{L} G}(s, \theta)) \mid(s, \theta) \in I \times[0,2 \pi)\}$. By the similar calculations to the case 2, we have the following proposition.

Proposition 10.2 For $\boldsymbol{v}_{0} \in S_{+}^{2}$, we have the following:

(1) $h_{\boldsymbol{v}_{0}}^{\prime}\left(s_{0}\right)=0$ if and only if there exists $\theta_{0} \in[-\pi . \pi)$ such that $\boldsymbol{v}_{0}=\widetilde{\mathbb{L} \mathbb{G}}\left(s_{0}, \theta_{0}\right)$.

(2) $h_{\boldsymbol{v}_{0}}^{\prime}\left(s_{0}\right)=h_{\boldsymbol{v}_{0}}^{\prime \prime}(s)=0$ if and only if there $\boldsymbol{v}_{0}=\widetilde{\mathbb{L} G}\left(s_{0}, \pm \pi / 2\right)$.

(3) $h_{\boldsymbol{v}_{0}}^{\prime}\left(s_{0}\right)=h_{\boldsymbol{v}_{0}}^{\prime \prime}(s)=h_{\boldsymbol{v}_{0}}^{\prime \prime \prime}\left(s_{0}\right)=0$ if and only if there $\boldsymbol{v}_{0}=\widetilde{\mathbb{L} \mathbb{G}}\left(s_{0}, \pm \pi / 2\right)$ and $\kappa_{2}\left(s_{0}\right)=0$.

(4) $h_{\boldsymbol{v}_{0}}^{\prime}\left(s_{0}\right)=h_{\boldsymbol{v}_{0}}^{\prime \prime}(s)=h_{\boldsymbol{v}_{0}}^{\prime \prime \prime}\left(s_{0}\right)=h_{\boldsymbol{v}_{0}}^{(4)}\left(s_{0}\right)=0$ if and only if there $\boldsymbol{v}_{0}=\widetilde{\mathbb{L} \mathbb{G}}\left(s_{0}, \pm \pi / 2\right), \kappa_{2}\left(s_{0}\right)=0$ and $\kappa_{2}^{\prime}\left(s_{0}\right)=0$.

Proof. By the calculations of fourth order derivatives of the lightcone height function $h_{\boldsymbol{v}}$, we can show the assertions similar way to the proof of Proposition 10.1. 


\subsection{Classifications of singularities}

By using the results of the above cases, we classify the singularities of the lightcone pedals as an application of the unfolding theory of functions. For a function $f(s)$, we say that $f$ has $A_{k^{-}}$ singularity at $s_{0}$ if $f^{(p)}\left(s_{0}\right)=0$ for all $1 \leq p \leq k$ and $f^{(k+1)}\left(s_{0}\right) \neq 0$. Let $F$ be an $r$-parameter unfolding of $f$ and $f$ has $A_{k}$-singularity $(k \geq 1)$ at $s_{0}$. We denote the $(k-1)$-jet of the partial derivative $\partial F / \partial x_{i}$ at $s_{0}$ as

$$
j^{(k-1)}\left(\frac{\partial F}{\partial x_{i}}\left(s, \boldsymbol{x}_{0}\right)\right)\left(s_{0}\right)=\sum_{j=1}^{k-1} \alpha_{j i}\left(s-s_{0}\right)^{j}, \quad(i=1, \cdots, r) .
$$

If the rank of $k \times r$ matrix $\left(\alpha_{0 i}, \alpha_{j i}\right)$ is $k(k \leq r)$, then $F$ is called a $\mathcal{R}$-versal unfolding of $f$, where $\alpha_{0 i}=\partial F / \partial x_{i}\left(s_{0}, \boldsymbol{x}_{0}\right)$.

Inspired by the propositions in the previous subsections, we define the following set:

$$
D_{F}^{\ell}=\left\{\boldsymbol{x} \in \mathbb{R}^{r} \mid \exists s \in \mathbb{R}, F(s, \boldsymbol{x})=\frac{\partial F}{\partial s}(s, \boldsymbol{x})=\cdots=\frac{\partial^{\ell} F}{\partial s^{\ell}}(s, \boldsymbol{x})=0\right\},
$$

which is called a discriminant set of order $\ell$. Then $D_{F}^{1}=D_{F}$ and $D_{F}^{2}$ is the set of singular points of $D_{F}$.

Therefore, $D_{H}=D_{H}^{1}=\mathbb{L} \mathbb{P}\left(N_{1}(C)\left[\boldsymbol{n}^{T}\right]\right)$ and $D_{H}^{2}$ Let $F$ and $G$ be $r$-parameter unfoldings of $f(s)$ and $g(s)$, respectively. We say that $F$ and $G$ are $P$ - $\mathcal{R}$-equivalent if there exists a diffeomorphism germ $\Phi:\left(\mathbb{R} \times \mathbb{R}^{r},\left(s_{0}, \boldsymbol{x}_{0}\right)\right) \longrightarrow\left(\mathbb{R} \times \mathbb{R}^{r},\left(s_{0}^{\prime}, \boldsymbol{x}_{0}^{\prime}\right)\right)$ of the form $\Phi(s, \boldsymbol{x})=\left(\Phi_{1}(s, \boldsymbol{x}), \phi(\boldsymbol{x})\right)$ such that $G \circ \Phi=F$. In this case we can easily show that $\Phi\left(D_{F}^{\ell}\right)=D_{G}^{\ell}$. By definition, the $\mathrm{P}$ - $\mathcal{R}$-equivalence implies the $\mathrm{P}-\mathcal{K}$-equivalence. We have the following classification theorem of $\mathcal{R}$-versal unfoldings [2, Page 149, 6.6].

Theorem 10.3 Let $F:\left(\mathbb{R} \times \mathbb{R}^{r},\left(s_{0}, \boldsymbol{x}_{0}\right)\right) \longrightarrow \mathbb{R}$ be an $r$-parameter unfolding of $f$ which has $A_{k}$-singularity at $s_{0}$. Suppose $F$ is an $\mathcal{R}$-versal unfolding of $f$, then $F$ is $P$ - $\mathcal{R}$-equivalent to one of the following unfoldings:

(a) $k=1 ; \pm s^{2}+x_{1}$,

(b) $k=2 ; s^{3}+x_{1}+s x_{2}$,

(c) $k=3 ; \pm s^{4}+x_{1}+s x_{2}+s^{2} x_{3}$,

We have the following classification result as a corollary of the above theorem.

Corollary 10.4 Let $F:\left(\mathbb{R} \times \mathbb{R}^{r},\left(s_{0}, \boldsymbol{x}_{0}\right)\right) \longrightarrow \mathbb{R}$ be an $r$-parameter unfolding of $f$ which has $A_{k}$-singularity at $s_{0}$. Suppose $F$ is a versal unfolding of $f$, then we have the following assertions: (a) If $k=1$, then $D_{F}$ is diffeomorphic to $\{0\} \times \mathbb{R}^{r-1}$ and $D_{F}^{2}=\emptyset$.

(b) If $k=2$, then $D_{F}$ is diffeomorphic to $C(2,3) \times \mathbb{R}^{r-2}, D_{F}^{2}$ is diffeomorphic to $\{\mathbf{0}\} \times \mathbb{R}^{r-2}$ and $D_{F}^{3}=\emptyset$.

(c) If $k=3$, then $D_{F}$ is diffeomorphic to $S W \times \mathbb{R}^{r-3}, D_{F}^{2}$ is diffeomorphic to $C(2,3,4) \times \mathbb{R}^{r-3}$, $D_{F}^{3}$ is diffeomorphic to $\{\mathbf{0}\} \times \mathbb{R}^{r-3}$ and $D_{F}^{4}=\emptyset$.

We remark that all of diffeomorphisms in the above assertions are diffeomorphism germs.

Here, we respectively call $C(2,3)=\left\{\left(x_{1}, x_{2}\right) \mid x_{1}=u^{2}, x_{2}=u^{3}\right\} \times \mathbb{R}$ a cuspdidaledge, $C(2,3,4)=\left\{\left(x_{1}, x_{2}, x_{3}\right) \mid x_{1}=u^{2}, x_{2}=u^{3}, x_{3}=u^{4}\right\}$ a $(2,3,4)$-cusp, $S W=\left\{\left(x_{1}, x_{2}, x_{3}\right) \mid\right.$ $\left.x_{1}=3 u^{4}+u^{2} v, x_{2}=4 u^{3}+2 u v, x_{3}=v\right\}$ a swallowtail (cf., Fig.1). 


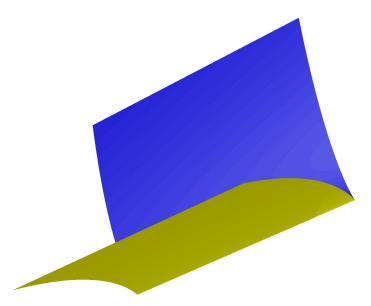

cuspidaledge

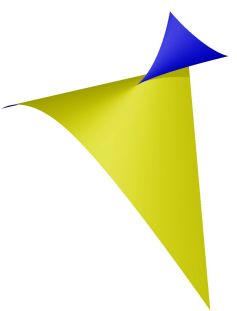

swallowtail

Fig. 1.

In order to apply the above theorem, we now define a function $\bar{H}: I \times S_{+}^{2} \times \mathbb{R} \longrightarrow \mathbb{R}$ by $\bar{H}(s, \boldsymbol{v}, r)=H(s, \boldsymbol{v})-r$. Let us consider the canonical diffeomorphism

$$
\Psi: S_{+}^{2} \times(\mathbb{R} \backslash\{0\}) \longrightarrow L C^{*}
$$

defined by $\Psi(\boldsymbol{v}, r)=r \boldsymbol{v}$. The we have $\widetilde{H}(s, \Psi(\boldsymbol{v}, r))=\langle\boldsymbol{\gamma}(s), \boldsymbol{v}\rangle-r=\bar{H}(s, \boldsymbol{v}, r)$. This means that $\widetilde{H}$ and $\bar{H}$ are P-R-equivalent. Here we have the following key proposition on $\bar{H}$.

Proposition 10.5 If $h_{\boldsymbol{v}_{0}}(s)$ has $A_{k}$-singularity $(k=1,2,3)$ at $s_{0}$, then $\bar{H}$ is a versal unfolding of $h_{\boldsymbol{v}_{0}}$.

Proof. We consider the local coordinate neighborhood $U_{1}^{+}=\left\{\boldsymbol{v}=\left(1, v_{1}, v_{2}, v_{3}\right) \in S_{+}^{2} \mid v_{1}>0\right\}$, where $v_{1}=\sqrt{1-v_{2}^{2}+v_{3}^{2}}$. We denote that $\gamma(s)=\left(x_{0}(s), x_{1}(s), x_{2}(s), x_{3}(s)\right)$ By definition, we have

$$
\bar{H}(s, \boldsymbol{v}, r)=-x_{0}(s)+x_{1}(s) \sqrt{1-v_{2}^{2}-v_{3}^{2}}+x_{2}(s) v_{2}+x_{3}(s) v_{3}-r .
$$

Thus we have

$$
\frac{\partial \bar{H}}{\partial v_{i}}(s, \boldsymbol{v}, r)=-\frac{v_{i}}{v_{1}} x_{1}(s)+x_{i}(s) \text { for } i=2,3
$$

and $\frac{\partial \bar{H}}{\partial r}(s, \boldsymbol{v}, r)=-1$. Thus, we have

$$
\frac{\partial^{2} \bar{H}}{\partial s \partial v_{i}}(s, \boldsymbol{v}, r)=-\frac{v_{i}}{v_{1}} x_{1}^{\prime}(s)+x_{i}^{\prime}(s) \text { and } \frac{\partial^{3} \bar{H}}{\partial s^{2} \partial v_{i}}(s, \boldsymbol{v}, r)=-\frac{v_{i}}{v_{1}} x_{1}^{\prime \prime}(s)+x_{i}^{\prime \prime}(s)
$$

It is enough to show that the rank of the following matrix is three:

$$
A=\left(\begin{array}{ccc}
\frac{v_{2}}{v_{1}} x_{1}\left(s_{0}\right)+x_{2}\left(s_{0}\right) & \frac{v_{3}}{v_{1}} x_{1}\left(s_{0}\right)+x_{3}\left(s_{0}\right) & -1 \\
\frac{v_{2}}{v_{1}} x_{1}^{\prime}\left(s_{0}\right)+x_{2}^{\prime}\left(s_{0}\right) & \frac{v_{3}}{v_{1}} x_{1}^{\prime}\left(s_{0}\right)+x_{3}^{\prime}\left(s_{0}\right) & 0 \\
\frac{v_{2}}{v_{1}} x_{1}^{\prime \prime}\left(s_{0}\right)+x_{2}^{\prime \prime}\left(s_{0}\right) & \frac{v_{3}}{v_{1}} x_{1}^{\prime \prime}\left(s_{0}\right)+x_{3}^{\prime \prime}\left(s_{0}\right) & 0
\end{array}\right)
$$

We now assume that $\boldsymbol{n}^{T}=\boldsymbol{n}_{2}$ (i.e., the case 2). By a straightforward calculation we can show that

$$
\operatorname{det}\left(\begin{array}{cc}
\frac{v_{2}}{v_{1}} x_{1}^{\prime}\left(s_{0}\right)+x_{2}^{\prime}\left(s_{0}\right) & \frac{v_{3}}{v_{1}} x_{1}^{\prime}\left(s_{0}\right)+x_{3}^{\prime}\left(s_{0}\right) \\
\frac{v_{2}}{v_{1}} x_{1}^{\prime \prime}\left(s_{0}\right)+x_{2}^{\prime \prime}\left(s_{0}\right) & \frac{v_{3}}{v_{1}} x_{1}^{\prime \prime}\left(s_{0}\right)+x_{3}^{\prime \prime}\left(s_{0}\right)
\end{array}\right)=\left(v_{1}, v_{2}, v_{3}\right) \cdot\left(\bar{\gamma}^{\prime}\left(s_{0}\right) \times \bar{\gamma}^{\prime \prime}\left(s_{0}\right)\right)
$$


where $\bar{\gamma}(s)=\left(x_{1}(s), x_{2}(s), x_{3}(s)\right) \in \mathbb{R}^{3}, \boldsymbol{a} \cdot \boldsymbol{b}$ is the Euclidean scaler product and $\boldsymbol{a} \times \boldsymbol{b}$ is the Euclidean vector product of $\boldsymbol{a}, \boldsymbol{b} \in \mathbb{R}^{3}$. Here, $\left(v_{1}, v_{2}, v_{3}\right) \cdot\left(\bar{\gamma}^{\prime}\left(s_{0}\right) \times \bar{\gamma}^{\prime \prime}\left(s_{0}\right)\right)$ is the first component of $\boldsymbol{e}_{0} \wedge \boldsymbol{\gamma}^{\prime}\left(s_{0}\right) \wedge \boldsymbol{\gamma}^{\prime \prime}\left(s_{0}\right)$ for $\boldsymbol{e}_{0}=(1,0,0,0)$. By the proof of Proposition 10.1, $\boldsymbol{v}_{0}=$ $\ell_{0}\left(s_{0}, \pm \pi / 2\right)\left(\boldsymbol{n}_{1} \pm \boldsymbol{n}_{3}\right)\left(s_{0}\right)$, so that

$\boldsymbol{e}_{0} \wedge \boldsymbol{\gamma}^{\prime}\left(s_{0}\right) \wedge \boldsymbol{\gamma}^{\prime \prime}\left(s_{0}\right)=\ell_{0}\left(s_{0}, \pm \pi / 2\right)\left(\boldsymbol{n}_{1} \pm \boldsymbol{n}_{3}\right)\left(s_{0}\right) \wedge \boldsymbol{t}\left(s_{0}\right) \wedge\left(\kappa_{1} \boldsymbol{n}_{1}\right)\left(s_{0}\right)= \pm \ell_{0}\left(s_{0}, \pm \pi / 2\right)\left(\kappa_{1} \boldsymbol{n}^{T}\right)\left(s_{0}\right)$.

Since $\boldsymbol{n}^{T}$ is timelike, the first component of $\boldsymbol{n}^{T}\left(s_{0}\right)$ is not equal to zero. By the similar calculations to the above, we can show that the rank of the matrix $A$ is three for the case 3 (i.e., $\left.\boldsymbol{n}^{T}=\boldsymbol{n}_{3}\right)$. This completes the proof.

Since $\Psi\left(D_{\bar{H}}^{\ell}\right)=D_{\widetilde{H}}^{\ell}$, we have the following classification result.

Theorem 10.6 Let $\gamma: I \longrightarrow \mathbb{R}_{1}^{4}$ be a spacelike curve with $\kappa_{1}(s) \neq 0$.

For the case 1, the lightcone pedal is always non-singular. For the cases 2 or 3, the set of singular points of the lightcone pedal is $\{(s, \pm \pi / 2) \mid s \in I\}$. Moreover, we have the following classification:

(1) The germ of the image of the lightcone pedal $\mathbb{L} \mathbb{P}(I \times[-\pi, \pi])$ at $\mathbb{L} \mathbb{P}\left(s_{0}, \pm \pi / 2\right)$ is diffeomorphic to the cuspidaledge $C(2,3) \times \mathbb{R}$ if $\kappa_{2}\left(s_{0}\right) \neq 0$. In this case the critical value set of the lightcone pedal is locally diffeomorphic to a line.

(2) The germ of the image of the lightcone pedal $\mathbb{L} \mathbb{P}(I \times[-\pi, \pi])$ at $\mathbb{L} \mathbb{P}\left(s_{0}, \pm \pi / 2\right)$ is diffeomorphic to the swallowtail $S W$ if $\kappa_{2}\left(s_{0}\right)=0$ and $\kappa_{2}^{\prime}\left(s_{0}\right) \neq 0$. In this case the critical value set of the lightcone pedal is locally diffeomorphic to the $(2,3,4)$-cusp $C(2,3,4)$.

Proof. We remark that $\mathbb{L} \mathbb{P}(I \times[-\pi . \pi])$ is the wavefront set of $\mathscr{L}_{\widetilde{H}}\left(\Sigma_{*}(\widetilde{H})\right)$. By definition, we have $\mathbb{L} \mathbb{P}(I \times[-\pi . \pi])=D_{\widetilde{H}}=\Psi\left(D_{\bar{H}}\right)$.

We now consider the cases 2 or 3 . By Proposition 10.5, $\bar{H}$ is an $\mathbb{R}$-versal unfolding of $h_{\boldsymbol{v}_{0}}$ at $s_{0}$ if $h_{\boldsymbol{v}_{0}}$ has $A_{k^{-}}$-singularity for $k=1,2,3$. By Propositions 10.1 and $10.2, h_{\boldsymbol{v}_{0}}$ has $A_{2}$-singularity at $s_{0}$ for $\boldsymbol{v}_{0}=\widetilde{\mathbb{L} G}\left(s_{0}, \pm \pi / 2\right)$ if $\kappa_{2}\left(s_{0}\right) \neq 0$. In this case, by Corollary 10.4, the germ of the image of the lightcone pedal $\mathbb{L} \mathbb{P}(I \times[-\pi, \pi])$ at $\mathbb{L} \mathbb{P}\left(s_{0}, \pm \pi / 2\right)$ is diffeomorphic to the cuspidaledge $C(2,3) \times \mathbb{R}$. Moreover, $h_{\boldsymbol{v}_{0}}$ has $A_{3}$-singularity at $s_{0}$ for $\boldsymbol{v}_{0}=\widetilde{\mathbb{L} G}\left(s_{0}, \pm \pi / 2\right)$ if $\kappa_{2}\left(s_{0}\right)=0$ and $\kappa_{2}^{\prime}\left(s_{0}\right) \neq 0$. In this case, the germ of the image of the lightcone pedal $\mathbb{L} \mathbb{P}(I \times[-\pi, \pi])$ at $\mathbb{L} \mathbb{P}\left(s_{0}, \pm \pi / 2\right)$ is diffeomorphic to the swallowtail $S W$. By Corollary 10.4, the critical value set of the lightcone pedal is locally diffeomorphic to the line and the $(2,3,4)$-cusp $C(2,3,4)$ respectively. This completes the proof.

\section{Submanifolds in Euclidean space or Hyperbolic space}

In this section we consider submanifolds in Euclidean space and Hyperbolic space as special cases as the previous results.

\subsection{Submanifolds in Euclidean space}

Let $\mathbb{R}_{0}^{n}$ be the Euclidean space which is given by $x_{0}=0$ for $\boldsymbol{x}=\left(x_{0}, x_{1}, \ldots, x_{n}\right)$. Consider an embedding $\boldsymbol{X}: U \longrightarrow \mathbb{R}_{0}^{n}$, where $U \subset \mathbb{R}^{s}$ is an open set. In this case we can adopt 
$\boldsymbol{n}^{T}=\boldsymbol{e}_{0}=(1,0, \ldots, 0)$ as a future directed timelike unit normal vector field along $M=\boldsymbol{X}(U)$ in $\mathbb{R}_{1}^{n+1}$. In this case $N_{1}(M)\left[\boldsymbol{n}^{T}\right]=N_{1}(M)\left[\boldsymbol{e}_{0}\right]$ is the unit normal bundle $N_{1}^{e}(M)$ of $M$ in $\mathbb{R}_{0}^{n}$ in the Euclidean sense. Therefore, the lightcone Gauss map $\widetilde{\mathbb{L} G}\left(\boldsymbol{n}^{T}\right)$ is given by $\widetilde{\mathbb{L} G}\left(\boldsymbol{n}^{T}\right)(p, \boldsymbol{\xi})=$ $\boldsymbol{e}_{0}+\boldsymbol{\xi}=\boldsymbol{e}_{0}+\mathbb{G}(p, \boldsymbol{\xi})$, where $\mathbb{G}: N_{1}^{e}(M) \longrightarrow S^{n-1}$ is the Gauss map of the unit normal bundle $N_{1}^{e}(M)$ defined by $\mathbb{G}(p, \boldsymbol{\xi})=\boldsymbol{\xi}[3]$. Since $\boldsymbol{e}_{0}$ is a constant vector, we have $d_{(p, \boldsymbol{\xi})} \widetilde{\mathbb{L} G}\left(\boldsymbol{n}^{T}\right)=d_{(p, \boldsymbol{\xi})} \mathbb{G}$, so that we have

$$
\kappa_{i}\left(\boldsymbol{n}^{T}\right)(p, \boldsymbol{\xi})=\kappa_{i}\left(\boldsymbol{e}_{0}\right)(p, \boldsymbol{\xi})=\kappa_{i}(p, \boldsymbol{\xi}),
$$

where $\kappa_{i}(p, \boldsymbol{\xi})(i=1, \ldots, s)$ are the eigenvalues of $-d_{(p, \boldsymbol{\xi})} \mathbb{G}$ belonging to the eigenvectors on $T_{p} M$, which are the principal curvatures of $M$ with respect to $\boldsymbol{\xi}$ in the Euclidean sense. The intersection of $\mathbb{R}_{0}^{n}$ with a lightlike hyperplane in $R_{0}^{n+1}$ is a hyperplane in $\mathbb{R}_{0}^{n}$. Therefore, the lightlike flat geometry of submanifolds in $\mathbb{R}_{0}^{n}$ is a geometry investigating the contact with hyperplanes of $\mathbb{R}_{0}^{n}$ which is called the flat geometry in Euclidean space. For example, let $\gamma: I \longrightarrow \mathbb{R}_{0}^{3}$ be a unit speed curve in Euclidean 3-space. It corresponds to the case 3) in $\S 10$. We choose $\boldsymbol{n}^{T}(u)=\boldsymbol{e}_{0}=(1,0,0,0)=\boldsymbol{n}_{3}(u)$, so that $\kappa_{1}(s)$ is the curvature of $\boldsymbol{\gamma}$ and $\kappa_{2}(s)$ is the torsion of $\boldsymbol{\gamma}$. Thus, Proposition 10.2 asserts that a singular point of the Gauss map corresponds to the point of the curve which has higher order contact with a plane in $\mathbb{R}_{0}^{3}$.

\subsection{Submanifolds in Hyperbolic space}

Let $\boldsymbol{X}: U \longrightarrow H^{n}(-1)$ be an immersion into the hyperbolic space. Then we adopt $\boldsymbol{n}^{T}(u)=$ $\boldsymbol{X}(u)$. In this case $N_{1}(M)\left[\boldsymbol{n}^{T}\right]$ is the unit normal bundle $N_{1}^{h}(M)$ of $M=\boldsymbol{X}(U)$ in $H^{n}(-1)$. Therefore, the lightcone Gauss image $\mathbb{L} \mathbb{G}\left(\boldsymbol{n}^{T}\right)$ is given by $\mathbb{L} \mathbb{G}\left(\boldsymbol{n}^{T}\right)(u, \boldsymbol{\xi})=\boldsymbol{X}(u)+\boldsymbol{\xi}$. We remember that

$$
C M_{2}\left(\boldsymbol{n}^{T} ; r\right)=\overline{\boldsymbol{X}}\left(\boldsymbol{n}^{T}, \boldsymbol{N}^{S} ; r\right)\left(U \times S^{k-2}\right)=\left\{\boldsymbol{X}(u)+\boldsymbol{N}^{S}(u, \mu) \mid(u, \mu) \in U \times S^{k-2}\right\} .
$$

For sufficiently small $1>r>0$, there exists $\theta \in \mathbb{R}$ such that $\tanh \theta=r$. We now define a mapping

$$
\Psi_{h}: C M_{2}\left(\boldsymbol{n}^{T} ; r\right) \longrightarrow H^{n}(-1)
$$

by $\Psi_{h}\left(\boldsymbol{X}(u)+r \boldsymbol{N}^{S}(u, \mu)\right)=\cosh \theta\left(\boldsymbol{X}(u)+r \boldsymbol{N}^{S}(u, \mu)\right)=\cosh \theta \boldsymbol{X}(u)+\sinh \theta \boldsymbol{N}^{S}(u, \mu)$. We remark that $\Psi_{h}$ is an embedding and the image is the hyperbolic canal hypersurface $C_{h}(M ; \theta)$ of $M$ defined in [9]. In [9] the horospherical hypersurface of $M$ was defined to be a mapping

$$
H S_{\boldsymbol{X}}: U \times S^{k-2} \longrightarrow L C^{*} ; H S_{\boldsymbol{X}}(u, \mu)=\boldsymbol{X}(u)+\boldsymbol{N}^{S}(u, \mu),
$$

which is nothing but the lightcone Gauss image $\mathbb{L} \mathbb{G}: U \times S^{k-2} \longrightarrow L C^{*}$.

We now define a mapping $\Phi: L C^{*} \longrightarrow L C^{*}$ by $\Phi(\boldsymbol{v})=\frac{-1}{v_{0}^{2}} \boldsymbol{v}$, wehre $\boldsymbol{v}=\left(v_{0}, v_{1}, \ldots, v_{n}\right)$. Then we have $\Phi \circ \Phi=1_{L C^{*}}$, so that $\Phi$ is a diffeomorphism. If we denote that $\boldsymbol{X}(u)+\boldsymbol{N}(u, \mu)=$ $\left(\ell_{0}(u, \mu), \ell_{1}(u, \mu), \ldots, \ell_{n}(u, \mu)\right)$, then we have

$$
\widetilde{\mathbb{L} \mathbb{G}}(u, \mu)=\frac{1}{\ell_{0}(u, \mu)}\left(\boldsymbol{X}(u)+\boldsymbol{N}^{S}(u, \mu)\right) \text { and }\langle\boldsymbol{X}(u), \widetilde{\mathbb{L} \mathbb{G}}(u, \mu)\rangle=\frac{-1}{\ell_{0}(u, \mu)} .
$$

It follows that

$$
\mathbb{L} \mathbb{P}(u, \mu)=\langle\boldsymbol{X}(u), \widetilde{\mathbb{L} G}(u, \mu)\rangle \widetilde{\mathbb{L} \mathbb{G}}(u, \mu)=\Phi \circ \mathbb{L} \mathbb{G}(u, \mu)=\Phi \circ H S_{\boldsymbol{X}}(u, \mu) .
$$

Therefore, we have [9, Theorem 5.6] as a special case of Theorem 8.6. In this case, the lightlike flat geometry is called the horospherical geometry in Hyperbolic space [6, 9]. 


\section{References}

[1] V. I. Arnol'd, S. M. Gusein-Zade and A. N. Varchenko, Singularities of Differentiable Maps vol. I. Birkhäuser, 1986.

[2] J. W. Bruce, P. J. Giblin, Curves and singularities(2nd.ed.), Cambridge Univ. Press (1992).

[3] S. -S. Chern and R. K. Lashof, On the total curvature of immersed manifolds, Amer. J. Math., 79 (1957), 306-318

[4] M. Golubitsky and V. Guillemin, Contact equivalence for Lagrangian manifold, Adv. Math. 15 (1975), 375-387.

[5] V.V. Goryunov, Projections of generic surfaces with boundaries. Theory of singularities and its applications, 157-200, Adv. Soviet Math., 1, Amer. Math. Soc., Providence, RI (1990).

[6] S. Izumiya, D. Pei and T. Sano, Singularities of hyperbolic Gauss maps, Proc. London Math. Soc., vol. 86 (2003), 485-512

[7] S. Izumiya, D. Pei and M.C. Romero Fuster, The lightcone Gauss map of a spacelike surface in Minkowski 4-space, Asian J. Math., vol. 8 (2004), 511-530.

[8] S. Izumiya, D. Pei and M.C. Romero Fuster, Umbilicity of space-like submanifolds of Minkowski space, Proc. Roy. Soc. Edinburgh Sect. A 134 (2004), 375-387.

[9] S. Izumiya, D-H. Pei, M.C. Romero Fuster and M. Takahashi, The horospherical geometry of submanifolds in hyperbolic space, J. London Math. Soc. 71 (2005), 779-800.

[10] S. Izumiya, M. Kossowski, D. Pei and M.C. Romero Fuster, Singularities of lightlike hypersurfaces in Minkowski 4-space, Tohoku Math. J. (2) 58 (2006), 71-88.

[11] S. Izumiya and M. C. Romero Fuster, The lightlike flat geometry on spacelike submanifolds of codimension two in Minkowski space, Selecta Mathematica (NS) 13 (2007), 23-55.

[12] S. Izumiya, Total lightcone curvatures of spacelike submanifolds in Lorentz-Minkowski space, preprint (2012)

[13] S. Izumiya and T. Sato, Lightlike hypersurfaces along spacelike submanifolds in Minkowski space-time, Journal of Geometry and Physics 71 (2013), 30-52.

[14] M. Kossowski, The $S^{2}$-valued Gauss maps and split total curvature of space-like codimension-2 surfaces in Minkowski space, J. London Math. Soc.(2) 40 (1989), 179-192.

[15] J. Martinet, Singularities of Smooth Functions and Maps, London Math. Soc. Lecture Note Ser. 58, Cambridge Univ. Press, Cambridge-New York, 1982.

[16] J. A. Montaldi, On contact between submanifolds, Michigan Math. J., 33 (1986), 81-85.

[17] B. O’Neill, Semi-Riemannian Geometry, Academic Press, New York, 1983.

[18] V. M. Zakalyukin, Lagrangian and Legendrian singularities, Funct. Anal. Appl. (1976), 2331. V. M. Zakalyukin, Reconstructions of fronts and caustics depending one parameter and versality of mappings, J. Sov. Math. 27 (1984), 2713-2735. 
SHYUICHI IZUMIYA

Department of Mathematics

HOKKAIDO UNIVERSITY

SAPPORO 060-0810

JAPAN

E-mail address: izumiya@math.sci.hokudai.ac.jp

MASAKI KASEDOU

DePartment of MATHEMATiCs

HOKKAIDO UNIVERSITY

SAPPORO 060-0810

JAPAN

E-mail address: kasedou@math.sci.hokudai.ac.jp 